\title{
On achieving network throughput demand in cognitive radio-based home area networks
}

\author{
Mohd Adib Sarijari ${ }^{1,2^{*}}$, Mohd Sharil Abdullah², Gerard JM Janssen ${ }^{1}$ and Alle-Jan van der Veen ${ }^{1}$
}

\begin{abstract}
The growing number of wireless devices for in-house use is causing a more intense use of the spectrum to satisfy the required quality-of-service such as throughput. This has contributed to spectrum scarcity and interference problems particularly in home area networks (HAN). Cognitive radio (CR) has been recognized as one of the most important technologies which could solve these problems and sustainably meeting the required communication demands by intelligently exploiting temporarily unused spectrum, including licensed spectrum. In this paper, we propose a throughput demand-based cognitive radio solution for home area networks (TD-CRHAN) which aims at effectively and efficiently meet the ever-increasing throughput demand in HAN communication. It is shown numerically and by simulations that a TD-CRHAN can satisfy the requested throughput from the network devices and has high utilization of the available throughput. The analysis further shows that, by setting the achievable throughput to be as close as possible to the total demanded throughput (instead of maximizing it), a TD-CRHAN is able to relax the tight cooperative spectrum sensing requirements which significantly improves cooperative spectrum sensing parameters, such as the local spectrum sensing time and the number of cooperative spectrum sensing devices. Finally, it is shown that these cooperative spectrum sensing parameters can be further improved when additional channels are available.
\end{abstract}

Keywords: Home area network communication; Throughput; Cognitive radio; Cooperative spectrum sensing

\section{Introduction}

A future home area network (HAN) is envisaged to consist of a large number of devices that support various applications including smart grid, security and safety systems, voice call, and video streaming. Most of these home devices are communicating based on various wireless networking technologies such as WiFi, ZigBee, and Bluetooth which typically operated in the already congested ISM licensed-free frequency band [1]. As these devices are located in a small physical space (i.e., limited by the size of the house) creating a dense HAN, they might interfere one another and causing a severe limitation to the quality of service (QoS) such as throughput. These issues are further aggravated in dense cities where the HAN also receives interference from neighboring HANs. Cognitive radio (CR) is seen as one of the most promising technologies to solve these problems and at the same

\footnotetext{
*Correspondence: m.a.b.sarijari@tudelft.nl

1 Faculty of Electrical Engineering, Mathematics and Computer Sciences, Delft University of Technology, 2628CD Delft, the Netherlands

${ }^{2}$ Faculty of Electrical Engineering, Universiti Teknologi Malaysia, 81310 UTM Johor Bahru, Johor, Malaysia
}

time fulfill the HAN's communication needs. CR technology enables the HAN devices to intelligently exploit idle spectrum including licensed spectrum for their communications, avoiding from being interfered as well as causing interference to others (in particular, the incumbent user).

A key component of CR-based networks is spectrum sensing, i.e., to reliably identify temporarily unused spectrum which is then exploited. Many existing works on throughput-based spectrum sensing focus on maximizing the achievable throughput. In [2], the maximum achievable throughput is obtained by optimizing the local spectrum sensing time, subject to a certain level of spectrum owner protection. The work in [3] incorporates the parameters from spectrum sensing (i.e., sensing time and number of cooperating devices decided the channel is occupied) and spectrum access (i.e., transmission probability) and optimizes those parameters to yield the maximum throughput for a given spectrum set. Further, in [4], the optimal sensing order for the channels

\section{Springer}

(C) 2015 Sarijari et al. Open Access This article is distributed under the terms of the Creative Commons Attribution 4.0 International License (http://creativecommons.org/licenses/by/4.0/), which permits unrestricted use, distribution, and reproduction in any medium, provided you give appropriate credit to the original author(s) and the source, provide a link to the Creative Commons license, and indicate if changes were made. 
is determined based its occupancy history, i.e., by correlating the channel availability statistics across time and frequency, in order to maximized the total achievable throughput. In addition, in our previous work [5] and in [6], throughput maximization is achieved by determining the optimal local spectrum sensing time, number of cooperating nodes and fusion strategy. However, aiming at maximizing the achievable network throughput leads to tight requirements on cooperative spectrum sensing parameters (e.g., spectrum sensing time and number of cooperating devices). On the other hand, in practice, every communication network has a certain demanded throughput; hence, a maximization of the achievable network throughput without taking into consideration the actual network's needs is inefficient. Throughout this paper, we refer to this throughput maximizationbased solution in spectrum sensing as the conventional case.

In this work, we propose a throughput demand-based cognitive radio communication for home area networks (TD-CRHAN), where, instead of maximizing the achievable throughput, the TD-CRHAN seeks to tightly satisfy the network throughput demand. To the best of our knowledge, this is the first work proposing such an objective for CR-based HAN communication. In the TDCRHAN, the optimal local spectrum sensing time and number of cooperating devices required for spectrum sensing are determined, and it is shown that these are significantly lower as compared to the values from the conventional scheme. In addition, by taking into consideration the total throughput demand in designing the CR-based HAN communication, the TD-CRHAN scheme is also able to determine the optimal number of channels needed for the HAN.

We mathematically model the proposed TD-CRHAN scheme and formulate a suitable optimization problem with corresponding constraints. In the derivations, we consider general expressions for the cooperative spectrum sensing performance parameters (i.e., cooperative probability of false alarm, and detection). This supports scenarios in which the signal-to-noise ratio (SNR) of the incumbent user is not the same at different sensing devices and supports more general fusion rules, not limited to OR and AND rules only. Note that most of the previous works consider the same incumbent signal strength at all sensing devices and/or only consider OR and AND rules $[2,5-7]$ in order to simplify the analytical models and derivations. Assuming the same SNR is not realistic, in particular for indoor environments, because the sensing devices will be located at various locations where for example, devices that are located near the window may receive a relatively strong incumbent user's signal while devices which are located further inside the house will experience a very low signal strength.
Finally, we thoroughly analyze the performance of the TD-CRHAN, numerically and through simulations, where we compare the performance with the conventional scheme, illustrate the impact of different parameter settings, and demonstrate the significant gains obtained from TD-CRHAN.

The remaining of this paper is organized as follows. Section 2 explains the proposed TD-CRHAN; Section 3 presents the derivation of the considered system model and the cooperative spectrum sensing, as well as the formulation of the problem and the proposed solution; the numerical analysis and the simulation results are presented in Sections 4 and 5, respectively; and the conclusions are in Section 6. A list of key symbols used in this paper is given in Table 1 .

Table 1 List of key symbols

\begin{tabular}{|c|c|}
\hline Symbol & Description \\
\hline D & Demanded throughput \\
\hline $\mathcal{H}_{0}$ & Hypothesis of the channel is idle \\
\hline $\mathcal{H}_{1}$ & Hypothesis of the channel is occupied \\
\hline$i$ & Channel's number \\
\hline 1 & Total active in-band channel in the cluster \\
\hline j & CR-based HAN device's number \\
\hline J & Total number of CR-based HAN devices in the HAN \\
\hline K & $\begin{array}{l}\text { Total number of devices that decides the sensed channel } \\
\text { is occupied }\end{array}$ \\
\hline L & $\begin{array}{l}\text { Total number of local spectrum sensing samples within a } \\
\text { sensing period }\end{array}$ \\
\hline N & $\begin{array}{l}\text { Total number of cooperating nodes for cooperative } \\
\text { spectrum sensing }\end{array}$ \\
\hline$P_{f}$ & Local probability of false alarm \\
\hline$P_{d}$ & Local probability of detection \\
\hline$Q_{f}$ & Cooperative probability of false alarm \\
\hline$Q_{d}$ & Cooperative probability of detection \\
\hline$R$ & Achievable throughput \\
\hline$u[$ [] & Received incumbent signal during the I-th sample \\
\hline $\mathrm{SNR}_{p}$ & SNR of the incumbent signal measured at the CR-device \\
\hline$T_{f}$ & Frame duration \\
\hline$T_{\text {CSS }}$ & Cooperative spectrum sensing time \\
\hline$T_{s}$ & Local spectrum sensing time \\
\hline$T_{s r}$ & Time duration for sending a single local sensing report \\
\hline$T_{t}$ & Data transmission time \\
\hline$w[$ l] & Noise signal during the $l$-th sample \\
\hline$\alpha$ & Fraction of data transmission time from the frame time \\
\hline$\varepsilon$ & $\begin{array}{l}\text { Difference between the achievable and the demanded } \\
\text { throughput }\end{array}$ \\
\hline$\tau$ & Sampling time \\
\hline$\gamma$ & Local spectrum sensing threshold \\
\hline
\end{tabular}




\section{Throughput demand-based cognitive radio home area network (TD-CRHAN) \\ 2.1 TD-CRHAN topology}

The proposed TD-CRHAN topology is based on a network of clustered CR devices as shown in Fig. 1. It consists of a HAN gateway (G), a cognitive HAN controller (C), a number of cognitive cluster heads $(\mathrm{CHs})$ and many $\mathrm{CR}$ based HAN devices. In such a network, the cognitive HAN controller is connected to the HAN gateway with a fixed connection while the $\mathrm{CHs}$ are linked to the cognitive HAN controller through wireless multi-hop links. The CHs are deployed such that each area of the house is covered. The communication among $\mathrm{CHs}$ is in a meshed manner. Each $\mathrm{CH}$ will form a network cluster. The CRbased HAN devices will need to connect to one of the clusters in order to communicate with or through the HAN network.

The functionalities of each network component are further described as follows.

The HAN gateway is the communication gateway for the HAN network to the outside world (i.e., the internet). Normally, the HAN gateway is connected to the internet service provider (ISP) for internet access through an Ethernet or Optical Fiber cable.
The other possible connection is via a wireless link, e.g., the WiMAX or LTE network.

The cognitive HAN controller is the device that is responsible to manage and coordinate the spectrum usage of the HAN. For this, the cognitive HAN controller needs to construct a spectrum map database for the particular HAN environment. This database consists of a list of channels that the $\mathrm{CH}$ can use in their cluster, and the condition of each channel, i.e., the statistics of the channel activities including channel utilization. It is constructed from the information fed by the $\mathrm{CHs}$ using for example the concept of MAC-layer sensing [8]. From this database, the cognitive HAN controller will provide the CHs with the channels that they could scan and utilize for their cluster. Therefore, the channels that the $\mathrm{CHs}$ are going to exploit are optimal and not random. In addition, in this way, the cognitive HAN controller also knows which channels are being utilized by which $\mathrm{CHs}$ and which are still unallocated. In this work, the channels that are allocated to the $\mathrm{CHs}$ are called in-band channels while the channels that are not allocated are called candidate channels. This concept is illustrated as in Fig. 2.

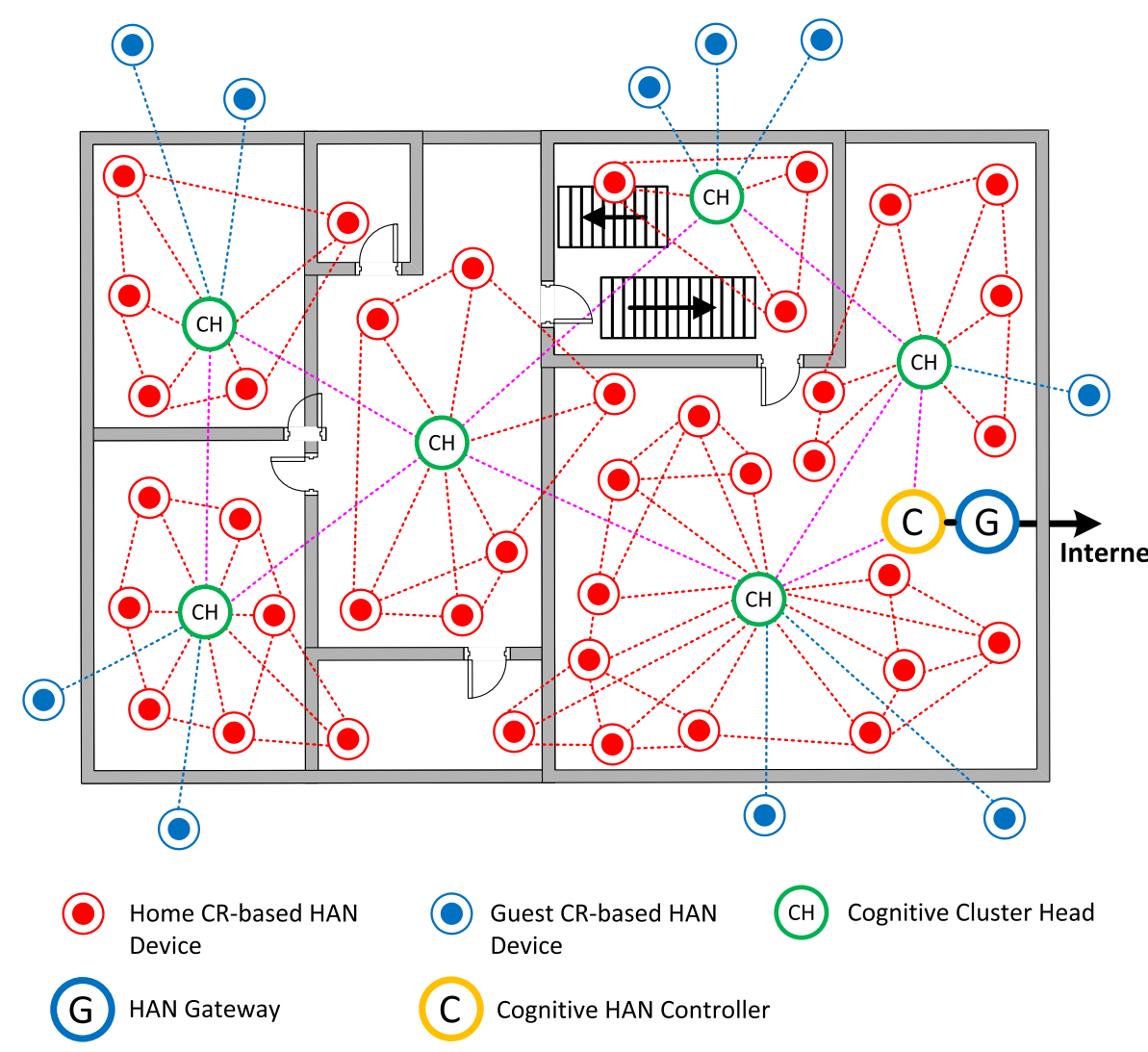

Fig. 1 The proposed network topology of TD-CRHAN 


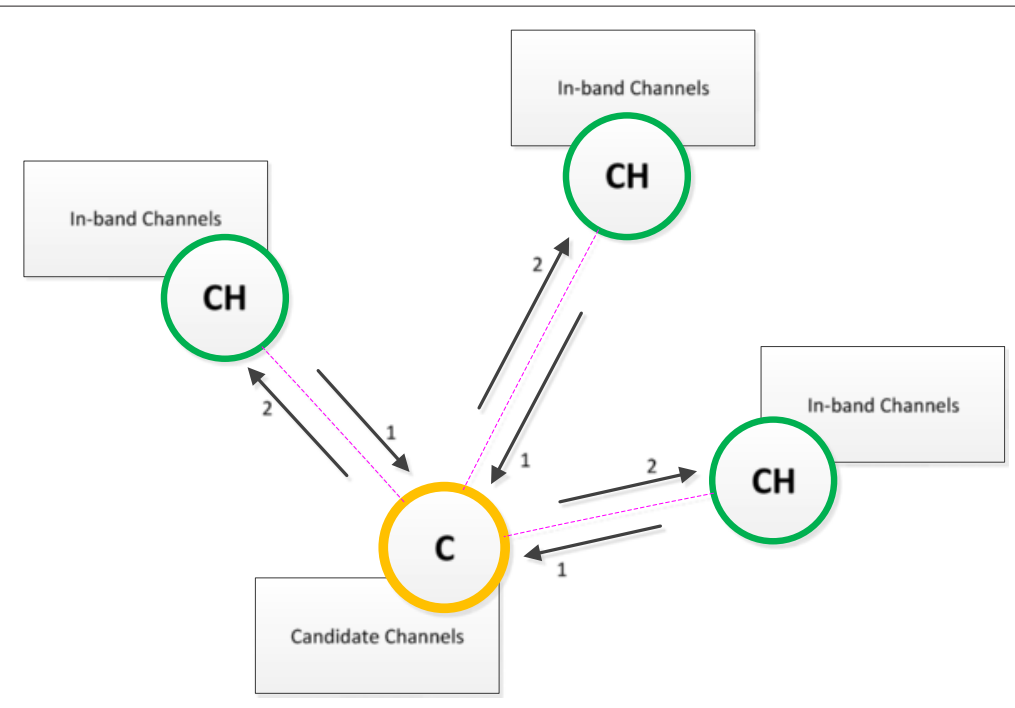

Fig. 2 The proposed spectrum management in TD-CRHAN

The cognitive cluster head (CH) is responsible to manage the usage of the cluster's in-band channels including sensing and access. A CH can request for more channels from the cognitive HAN controller if the current in-band channels are not enough to support its network cluster demand. Each $\mathrm{CH}$ will utilize different channels from the other $\mathrm{CHs}$ creating a distributed multi-channel network in the HAN. In addition, a $\mathrm{CH}$ is also responsible for selecting and grouping the CR-based HAN devices that are connected to it to perform the cooperative spectrum sensing (CSS) task. Besides, it also needs to schedule and distribute the selected and grouped CR-based HAN devices on when and where to sense, respectively. For CSS, a $\mathrm{CH}$ also acts as the fusion center where the local sensing results from the sensing devices will be reported to and the decision of spectrum availability will be made. Last but not least, from the CSS results, a $\mathrm{CH}$ is required to report the channel utilization and occupancy to the cognitive HAN controller periodically in order for the controller to construct and keep the spectrum map database up to date.

CR-based HAN devices are the devices that carry out various HAN applications including smart grid, security and safety, and home automation. These devices will connect to one of the clusters to get access and communicate with or through the HAN network. Besides performing the communication for its application, CR-based HAN devices also need to execute the spectrum sensing task. We consider two types of CR-based HAN devices: home and guest devices. Home devices are devices which belong to the HAN-owner, while guest devices do not belong to the HAN-owner. An example of a guest device is a neighbor's device which needs to off-load its traffic, e.g., due to congestion in its own HAN network. Another example is a device that passes through the house and wants to connect to the internet through the HAN network. For the home CR-based HAN devices, the communication topology within the cluster is in a mesh. However, the guest devices are only allowed to connect to the $\mathrm{CH}$.

\subsection{TD-CRHAN operation}

In TD-CRHAN, CR-based HAN devices need to be connected to one of the clusters in order to get access and communicate with or through the HAN network. For this, any cluster joining mechanism such as listed in [9] can be applied. One of the simple mechanisms is as employed in the IEEE 802.22 standard [10]. In this standard, the $\mathrm{CH}$ transmits a beacon at the beginning of each frame in each of the in-band channel. Alternatively, this beacon can be sent in one of the highest quality in-band channels. A CR-based HAN device will search for one of these beacons at its start-up and connect to the corresponding CH's cluster once the beacon is found. If the CR-based HAN device can hear beacons from multiple $\mathrm{CHs}$, it may choose to join either one cluster based on for example the signal strength and/or the signal quality of the received beacons [9].

Figure 3 illustrates the TD-CRHAN operation for one network cluster. In a TD-CRHAN, the bandwidth of the cluster is adaptable, it can be expanded or shrunk depending on the total throughput demand of the network cluster. In the example in the figure, at time $t_{0}$, the cluster only uses one in-band channel i.e., channel $B$. When the cluster needs more bandwidth, i.e., at time $t_{1}$ and $t_{4}$, for example 


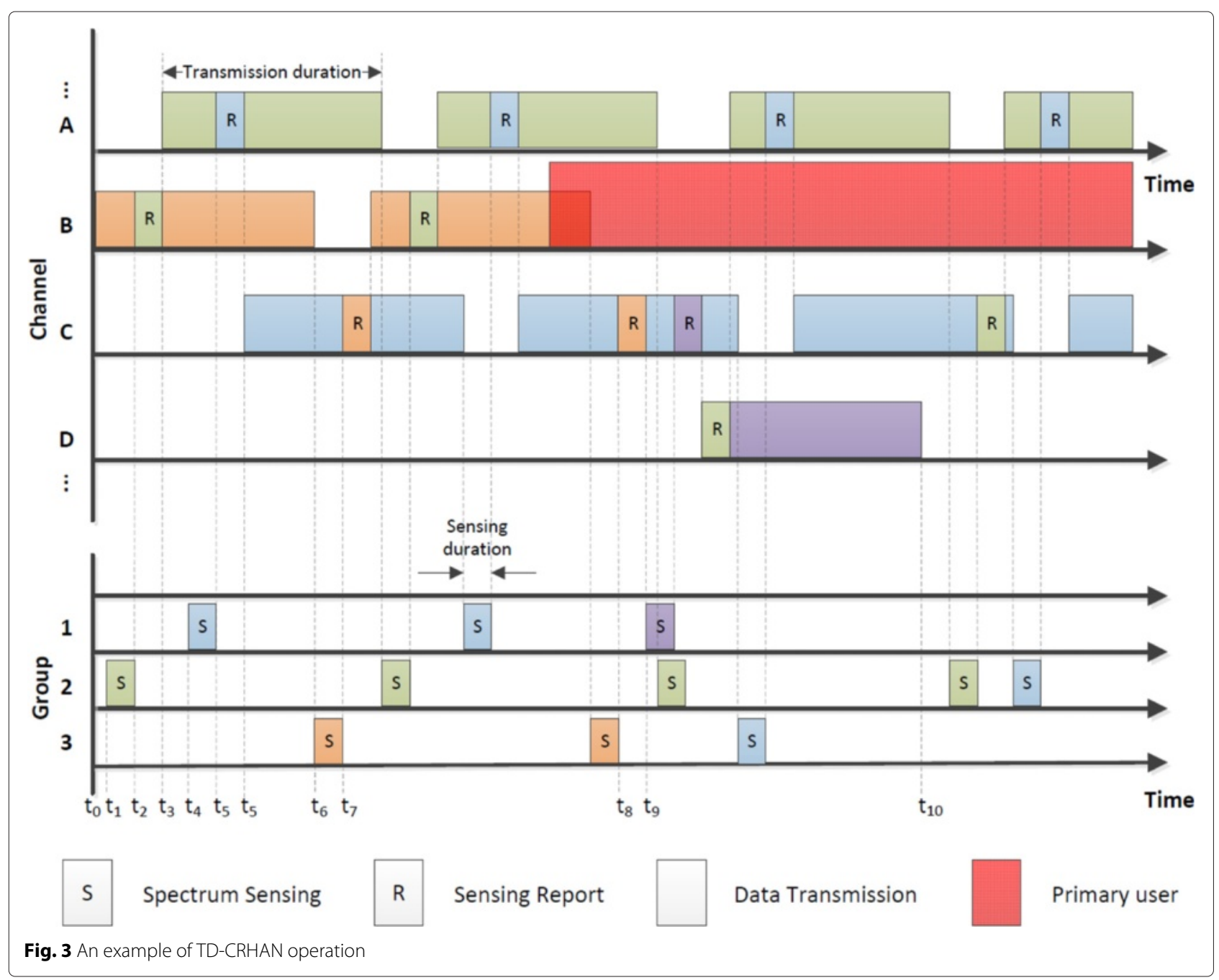

due to a new connected device, the number of in-band channels is increased to two channels with the addition of channel $A$, and to three channels with the addition of channel $C$, respectively. The additional in-band channels are obtained from the pool of candidate channels at the cognitive HAN controller. This process is illustrated by the arrows labeled "2" in Fig. 2. The cognitive HAN controller will provide the cluster with the best candidate channels it has. These channels will be passed on to the $\mathrm{CH}$.

In addition, at time $t_{9}$, the cluster shrinks its bandwidth by releasing one of its in-band channels that is channel $D$ due to a decrease in the network demand, e.g., due to a device leaving the cluster. The released channel is selected from the lowest quality channels among the in-band channels. This channel will be returned to the cognitive HAN controller and becomes a candidate channel that can be used by other clusters. This process is illustrated by the arrows labeled "1" in Fig. 2.

During typical CR operation, spectrum sensing will be executed first before any channel can be used for data transmission. In this work, the CSS method is considered. Therefore, the sensing operations will consist of spectrum sensing and reporting segments. For this, the CR-based devices will be grouped together forming multiple spectrum sensing groups in the cluster. For instance, in Fig. 3, three sensing groups are formed: groups 1,2 , and 3 . The $\mathrm{CH}$ will schedule and distribute the spectrum sensing tasks among these groups. In doing so, the $\mathrm{CH}$ has to ensure as much as possible that the group which is scheduled for sensing does not have any group member involved in active communication during this sensing period.

The $\mathrm{CH}$ also acts as the CSS's fusion center. Unlike in conventional CSS where the sensing results are transmitted either at the same sensed channels as in $[5,6]$ or by using a dedicated common control channel as in $[3,11]$, in TD-CRHAN, the sensing results are transmitted in one of the active transmission slots of the in-band channels as shown in Fig. 3. For this, the $\mathrm{CH}$ will inform the sensing groups on which channel the sensing reports should be transmitted and when. This information can be broadcasted by the $\mathrm{CH}$ through the beacons. In this way, 
the sensing reporting transmission will not interfere with the incumbent user of the channel, and the quality of the reporting channels are also ensured. Note that the sensing report information is very crucial, hence it needs to be highly reliable [12]. In case the dedicated common control channel is used, a dedicated channel will be required and the reporting transmission could cause this channel to be congested, and thus it may become the bottleneck of the network [11].

If the CSS results show that a channel is highly occupied (often busy), the $\mathrm{CH}$ will withdraw this channel from its in-band channels' list and return it to the HAN gateway. In the meantime, the $\mathrm{CH}$ can request for an additional inband channel from the HAN controller to overcome the throughput degradation due to this highly occupied inband channel. This scenario is illustrated at times $t_{8}$ and $t_{9}$ in Fig. 3 where the returned channel is channel $B$ and the new channel is channel $D$, respectively. In this example, the channel is returned to the HAN controller after one time it is sensed to be occupied.

In the next sections, we consider schemes to satisfy the TD-CRHAN network throughput demand with high resource (available throughput) utilization, and we determine the optimal local spectrum sensing time, the number of cooperating sensing devices and the number of active in-band channels needed.

\section{System model}

A simple network model (one cluster) of the proposed TD-CRHAN network is shown in Fig. 4. It consists of a HAN gateway (G), a cognitive HAN controller (C), a cluster head $(\mathrm{CH})$, and $J$ CR-based HAN devices $\mathrm{n}$ as $j, j=1,2, \ldots, J$. Every CR-based HAN device is equipped with a half-duplex radio that can be tuned to any combination of $I$ channels for data transmission and reception. This can be done by using, for example, the noncontiguous OFDM (NC-OFDM) technology [13]. Besides data communication, each CR-based HAN device is also able to perform a narrow-band spectrum sensing in which the sensing bandwidth is equal to the bandwidth of a single channel.

\subsection{Cooperative spectrum sensing}

In CSS, each cooperating CR-based HAN device will periodically sample the spectrum and send its local spectrum sensing result to a fusion center (in our case, this is the $\mathrm{CH})$. The $\mathrm{CH}$ will combine these local spectrum sensing results using a certain fusion strategy to make the final decision on whether the sensed spectrum is idle or not. In this work, a hard-fusion strategy is considered in which each cooperating CR-based HAN device makes a local decision and sends only this decision to the $\mathrm{CH}$. The local decision is a binary hypothesis test: decide whether the sensed channel is idle, given by hypothesis $\mathcal{H}_{0}$, or occupied, given by hypothesis $\mathcal{H}_{1}$. Each of the spectrumsamples observed by a CR-based HAN device can be modeled as

$$
x[l]= \begin{cases}w[l] & : \mathcal{H}_{0} \\ u[l]+w[l] & : \mathcal{H}_{1}\end{cases}
$$

where $l=1,2, \ldots, L$. Here, $L$ is the total number of observation samples made by a CR-based HAN device

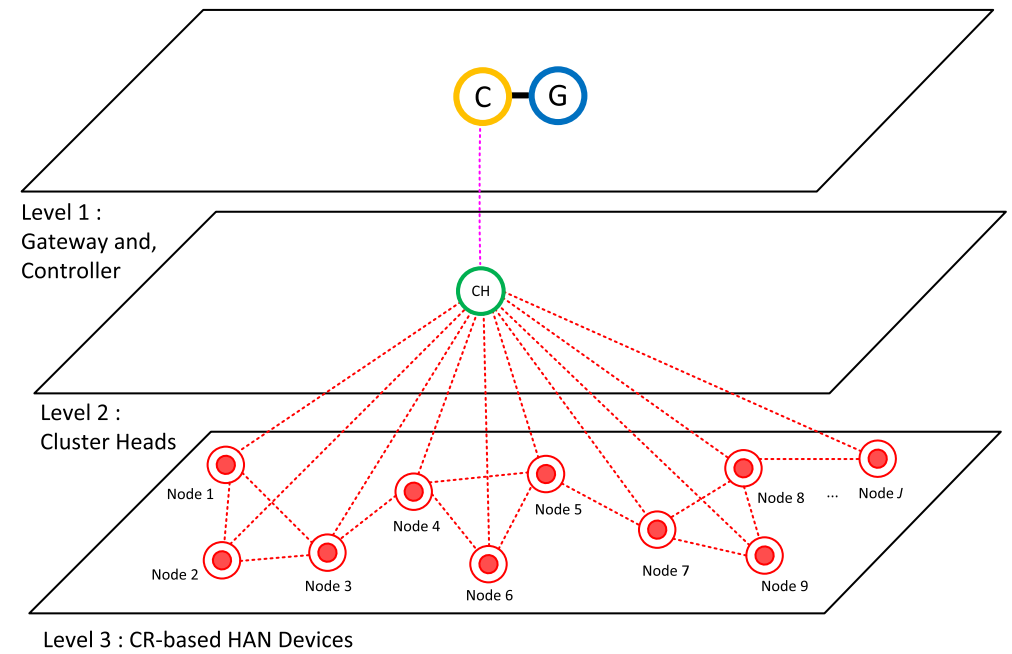

CR-based HAN Device

Cognitive Cluster Head

(G) HAN Gateway

Cognitive HAN Controller

Fig. 4 Network model diagram for a single TD-CRHAN cluster 
within the local spectrum sensing period $T_{s}$ such that $L=T_{s} / \tau$, where $\tau$ is the sampling period. We assume that the Nyquist sampling condition holds, i.e., $\tau$ is at least one over twice the channel bandwidth. Further, $u[l]$ is the received incumbent signal and $w[l]$ is the additive noise signal, during the $l$-th sample. $u[l]$ is given by $u[l]=s[l] * h[l]$, with $s[l]$ is the transmitted incumbent signal and $h[l]$ is the impact of Rayleigh fading channel. Note that $u[l]$ does not contain the impact of additive noise but the additive noise component is taken into account in $w[l]$. Both $w[l]$ and $u[l]$ are assumed to be independent and identically distributed (i.i.d.) random processes with zero mean and variance $\sigma_{w}^{2}$ and $\sigma_{u}^{2}$, respectively. We consider additive white Gaussian noise (AWGN) for $w[l]$ and a random signal with a Gaussian distribution for $u[l]$.

In this paper, energy detection is considered for spectrum sensing. The received power is estimated as

$$
\hat{E}=\frac{1}{L} \sum_{l=1}^{L} x^{2}[l]
$$

$\hat{E}$ is the output of the energy detector which is used as input for a binary hypothesis test of the CR-based HAN device. In the test, $\hat{E}$ is compared to a predefined threshold $\gamma$ to decide on hypothesis $\mathcal{H}_{0}$ or $\mathcal{H}_{1}$. The performance of this test is characterized by two metrics: the probability of detection $\left(P_{d}\right)$ and the probability of false alarm $\left(P_{f}\right)$. The probability that a CR-based HAN device decides that the channel is occupied (i.e., $\hat{E}>\gamma$ ) under $\mathcal{H}_{1}$ is given by

$$
P_{d}=P\left(\hat{E}>\gamma \mid \mathcal{H}_{1}\right)
$$

while the probability that a CR-based HAN device decides that the channel is occupied under $\mathcal{H}_{0}$ is

$$
P_{f}=P\left(\hat{E}>\gamma \mid \mathcal{H}_{0}\right)
$$

From $[2,5]$, for a targeted probability of detection $\bar{P}_{d}$, the corresponding probability of false alarm $P_{f}$ can be expressed as

$P_{f}\left(\bar{P}_{d}, \mathrm{SNR}_{p}, T_{s}\right)=\mathcal{Q}\left(\operatorname{SNR}_{p} \sqrt{\frac{T_{s}}{2 \tau}}+\mathcal{Q}^{-1}\left(\bar{P}_{d}\right) \sqrt{1+2 \mathrm{SNR}_{p}}\right)$

where $\mathcal{Q}(\cdot)$ denotes the usual Q-function (the tail probability of the standard normal distribution), and $\mathrm{SNR}_{p}:=$ $\sigma_{u}^{2} / \sigma_{w}^{2}$ is the signal-to-noise ratio of the incumbent user at the sensing device. Alternatively, if a target $\bar{P}_{f}$ needs to be achieved, the achievable $P_{d}$ can be formulated as $[2,5]$

$P_{d}\left(\bar{P}_{f}, \mathrm{SNR}_{p}, T_{s}\right)=\mathcal{Q}\left(\frac{1}{\sqrt{1+2 \mathrm{SNR}_{p}}}\left(\mathcal{Q}^{-1}\left(\bar{P}_{f}\right)-\mathrm{SNR}_{p} \sqrt{\frac{T_{s}}{2 \tau}}\right)\right)$.

Notice that any pair of $\bar{P}_{d}$ and $\bar{P}_{f}$ can be satisfied if the local spectrum sensing time $T_{s}$ is not restricted. From (5) or $(6)$ it follows that $[2,5]$

$$
\begin{aligned}
L=\frac{T_{s}\left(\mathrm{SNR}_{p}, \bar{P}_{d}, \bar{P}_{f}\right)}{\tau}= & \frac{2}{\operatorname{SNR}_{p}^{2}}\left(\mathcal{Q}^{-1}\left(\bar{P}_{f}\right)\right. \\
& \left.-\mathcal{Q}^{-1}\left(\bar{P}_{d}\right) \sqrt{1+2 \mathrm{SNR}_{p}}\right)^{2} .
\end{aligned}
$$

In this paper, we consider CSS with a hard-fusion strategy, wherein each cooperating CR-based HAN device sends its local decision to the $\mathrm{CH}$. The $\mathrm{CH}$ makes the final decision and decides $\mathcal{H}_{1}$ if at least $K$ out of $N$ cooperating CR-based HAN devices have decided that the channel is occupied; otherwise $\mathcal{H}_{0}$ will be decided. This strategy is known as the $K$-out-of- $N$ fusion rule. The cooperative probability of detection $Q_{d}$ and false alarm $Q_{f}$ under this fusion rule can be derived using the Poisson-Binomial distribution theorem as $[3,14,15]$

$$
\begin{aligned}
Q_{d} & =\sum_{k=K}^{N} \sum_{\mathcal{A}_{k}^{(a)} \in \mathcal{A}_{k}} \prod_{g \in \mathcal{A}_{k}^{(a)}} P_{d_{g}} \prod_{h \in\left\{\mathcal{N} \backslash \mathcal{A}_{k}^{(a)}\right\}}\left(1-P_{d_{h}}\right) \\
Q_{f} & =\sum_{k=K}^{N} \sum_{\mathcal{A}_{k}^{(a)} \in \mathcal{A}_{k}} \prod_{g \in \mathcal{A}_{k}^{(a)}} P_{f_{g}} \prod_{h \in\left\{\mathcal{N} \backslash \mathcal{A}_{k}^{(a)}\right\}}\left(1-P_{f_{h}}\right)
\end{aligned}
$$

where

$$
\begin{aligned}
& \mathcal{N}=\{1, \cdots, N\} \text { is a set consisting of all sensor } \\
& \text { indices, } \\
& \mathcal{A}_{k} \text { is a set consisting of all possible subsets of } k \\
& \text { elements of } \mathcal{N} \text {, representing the } k \text { out of } N \text { sensing } \\
& \text { devices that locally decide that the channel is } \\
& \text { occupied, } \\
& \mathcal{A}_{k}^{(a)} \in \mathcal{A}_{k} \text {, where a is an index, is one of the sets in } \\
& \mathcal{A}_{k}, \\
& g, h \in \mathcal{N} \text { are sensor indices. }
\end{aligned}
$$

There are three special cases in this fusion rule: 1 ) if $K=1$, the cooperative detection will become the OR combining rule, 2) if $K=N$, the fusion scheme follows the AND rule, and 3) if $K=\left\lceil\frac{N}{2}\right\rceil$, the decision is known as the majority rule. In addition, if $P_{d_{j}}$ (and $P_{f_{j}}$ ) are identical for all devices $j$ (i.e., $P_{d_{j}}=P_{d}$ and $P_{f_{j}}=P_{f}, \forall j$ ) which can be achieved for example by adapting the sensing time of each sensing device differently, then (8) and (9) can be simplified and formulated by using the normal Binomial distribution (instead of Poisson-Binomial), and become

$$
\begin{gathered}
Q_{d}=\sum_{k=K}^{N}\left(\begin{array}{l}
N \\
k
\end{array}\right) P_{d}^{k}\left(1-P_{d}\right)^{N-k} \\
Q_{f}=\sum_{k=K}^{N}\left(\begin{array}{c}
N \\
k
\end{array}\right) P_{f}^{k}\left(1-P_{f}\right)^{N-k}
\end{gathered}
$$

respectively, where $\left(\begin{array}{c}N \\ k\end{array}\right)$ is called the Binomial coefficient. 
In CR, $Q_{d}$ reflects the quality of protection of the band-owner and is determined by the regulator or the standardization body such as the IEEE (for example, in IEEE 802.22, $Q_{d}$ is required to be greater or equal to 0.9 , [10]). On the other hand, $Q_{f}$ is important for the CR devices (in our case, the CR-based HAN devices). A lower $Q_{f}$ will provide a higher opportunity for the CR-based HAN devices to access the spectrum and hence attain a higher network throughput. Note that IEEE 802.22, which is actually meant for rural area and large distance, is used as an example because it defines the spectrum sensing specifications (e.g., the probability of detection constraint) that are needed in this paper. Other newer standards like IEEE 802.11af and IEEE $802.15 .4 \mathrm{~m}$ would be more useful to home scenario, but there are no specifications given for the spectrum sensing because they are using the database method instead. In fact, the spectrum sensing parameters (e.g., $Q_{d} \geq 0.9$ constraint) used in the numerical and simulation in this paper are as examples and they can be changed to the desired values.

\subsection{Problem formulation}

Figure 5 shows the timing diagram of a single channel operation where the sensing-transmit task alternates in time. In this figure, $T_{f}$ is the time duration of a frame which is a constant, and it comprises two sub slots: a sub slot for the cooperative spectrum sensing $T_{c s s}$, and sub slot for data transmission $T_{t}$. The former is further divided into two parts, namely a time for local spectrum sensing $T_{s}$ and the time required to send the sensing result to the $\mathrm{CH} T_{s r}$. For reporting the local spectrum sensing result, a TDMA-based channel access scheme is employed, that is, the first CR-based device sends its decision in the first time slot, the second device in the second time slot, and so on (the same scheme is considered in [6]); thus, the total reporting time required for $N$ cooperating devices is $N \cdot T_{s r}$.
Note that we have

$$
\begin{aligned}
& T_{f}=T_{c s s}+T_{t} \\
& T_{c s s}=T_{s}+N T_{s r} .
\end{aligned}
$$

In addition, if the transmission uses rectangular signal pulses, then the maximum data rate for a single channel can be calculated as

$$
C=\frac{m W}{2} \text { (bit/second). }
$$

where $W$ is the null-to-null bandwidth of the channel, and $m=\log _{2}(M)(\mathrm{bit} / \mathrm{symbol})$ is the modulation order of the transmission when $M$ modulation levels are used.

In cognitive radio, each channel in the spectrum is periodically sensed and may only be utilized for data transmission if it is sensed idle, i.e., $\hat{E}<\gamma$. This may happen under both $\mathcal{H}_{0}$ and $\mathcal{H}_{1}$. Let the achievable throughput under scenario $\mathcal{H}_{0}$ be $R_{0}$. This throughput is smaller than $C$ by a factor $\left(1-Q_{f}\right)$, the probability that the channel is correctly detected as idle. Likewise, under $\mathcal{H}_{1}$, the achievable throughput $R_{1}$ is smaller than $C$ by a factor $\left(1-Q_{d}\right)$, which is the probability that the occupied channel is wrongly detected as idle. This probability is significant in case the incumbent signal is weak (e.g., due to the distance from the incumbent node to the CR network).

We also need to consider that for both scenarios the throughput is scaled by a factor $\alpha=T_{t} / T_{f}$, the fraction of time within a frame that data is transmitted. Using (12) and (13), we can write $\alpha$ as a function of the sensing time $T_{s}$ and number of sensing devices $N$ as

$$
\alpha\left(T_{s}, N\right)=1-\frac{\left(T_{s}+N T_{s r}\right)}{T_{f}} .
$$

Overall, this gives

$$
R_{0}=\alpha\left(T_{s}, N\right)\left(1-Q_{f}\right) C
$$

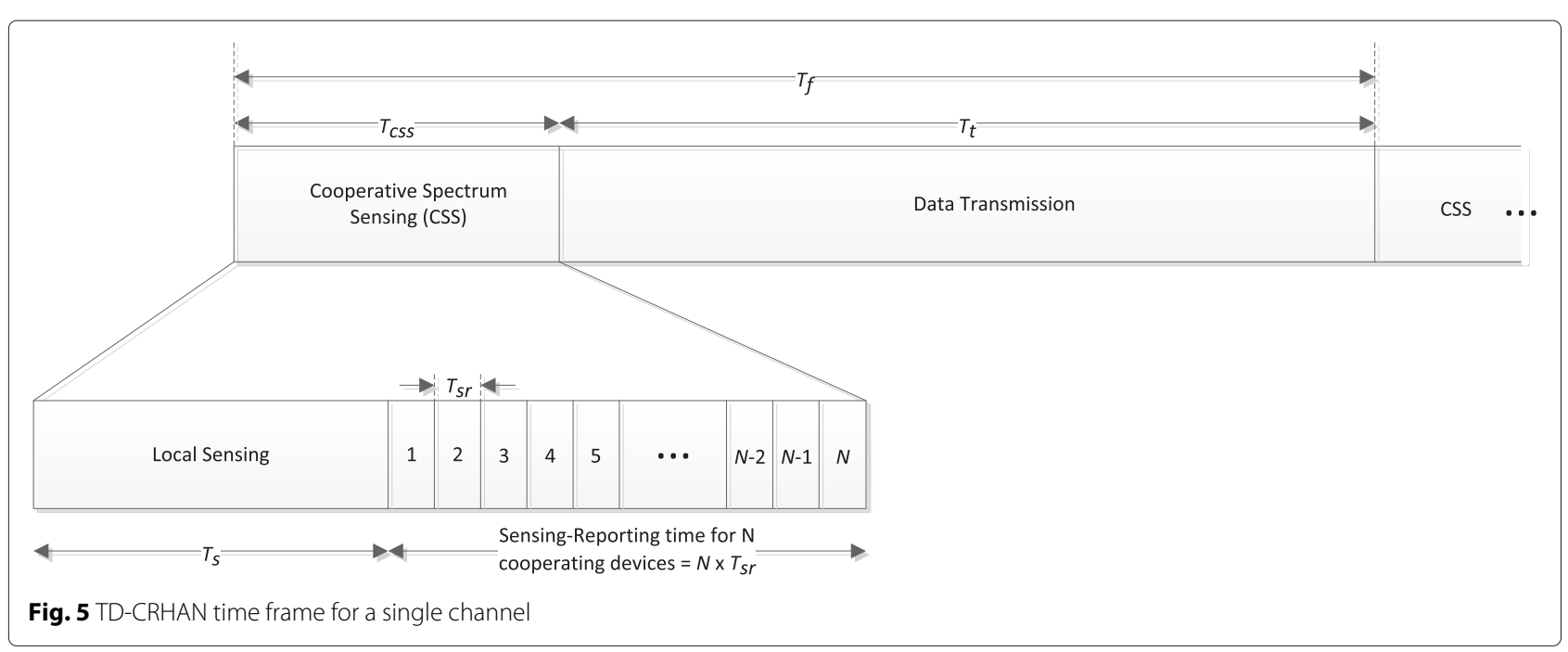




$$
R_{1}=\alpha\left(T_{s}, N\right)\left(1-Q_{d}\right) C,
$$

The achievable throughput of a single channel can then be formulated as

$$
R=P\left(\mathcal{H}_{0}\right) R_{0}+P\left(\mathcal{H}_{1}\right) R_{1}
$$

where $P\left(\mathcal{H}_{0}\right)$ and $P\left(\mathcal{H}_{1}\right)$ are the a priori probabilities that the channel is idle and occupied, respectively. These probabilities can be estimated before the CR network is deployed based on a long-term measurement or it can be measured online based on for example, the concept of MAC-layer sensing [8]. Substituting (18) in (16) and (17) gives

$$
R=\alpha\left(T_{s}, N\right) C\left(P\left(\mathcal{H}_{0}\right)\left(1-Q_{f}\right)+P\left(\mathcal{H}_{1}\right)\left(1-Q_{d}\right)\right) .
$$

Let $R^{(i)}$ be the achievable throughput for channel $i$, then the total achievable throughput for a cluster with $I$ simultaneously active channels can be calculated as

$$
\begin{aligned}
R_{t}= & \sum_{i=1}^{I} R^{(i)}=\sum_{i=1}^{I}\left[\alpha ( T _ { s } ^ { ( i ) } , N ^ { ( i ) } ) C \left(P\left(\mathcal{H}_{0}^{(i)}\right)\left(1-Q_{f}^{(i)}\right)\right.\right. \\
& \left.\left.+P\left(\mathcal{H}_{1}^{(i)}\right)\left(1-Q_{d}^{(i)}\right)\right)\right]
\end{aligned}
$$

Suppose that CR-based HAN device $j$ has a throughput demand of $d_{j}$. Then the total throughput demand in a cluster, coming of $J$ CR-based HAN devices, becomes

$$
D_{t}=\sum_{j=1}^{J} d_{j}
$$

This information can be acquired by the $\mathrm{CH}$ from each connected CR-based HAN device, for example at the time that the device is requesting to join the cluster, or updated by the CR-based HAN device to the $\mathrm{CH}$ whenever there is a change in its throughput demand.

Let $\varepsilon=R_{t}-D_{t}$ be the difference between $R_{t}$ and $D_{t}$. Using (19)-(21), we can write $\varepsilon$ as

$$
\begin{aligned}
\varepsilon(I, \alpha)= & \sum_{i=1}^{I}\left[\alpha ( T _ { s } ^ { ( i ) } , N ^ { ( i ) } ) C \left(P\left(\mathcal{H}_{0}^{(i)}\right)\left(1-Q_{f}^{(i)}\right)\right.\right. \\
& \left.\left.+P\left(\mathcal{H}_{1}^{(i)}\right)\left(1-Q_{d}^{(i)}\right)\right)\right]-D_{t}
\end{aligned}
$$

\subsection{Throughput demand-based CR communication}

It is important to ensure that the difference between $R_{t}$ and $D_{t}$ is as small as possible. A positive value of $\varepsilon$ means that the available throughput of the active channels in the cluster is underutilized while a negative value means that the QoS of the throughput demand is not fulfilled. Notice that in a TD-CRHAN, in case that a cluster's demand is higher than the capacity of a single channel i.e., $D_{t}>R_{t}$, the $\mathrm{CH}$ in the particular cluster should ask for additional channels from the cognitive HAN controller until the demand is met.

Theoretically, if the number of channels $I$ is unlimited, then the TD-CRHAN scheme can support any amount of throughput demand. With a higher number of channels, we can reduce $T_{s}$ and $N$ (c.f., (20)). However, activating more channels will consume more bandwidth. Hence, optimal values of $I, T_{s}$ and $N$, that can give the minimum $\varepsilon$ should be determined. This optimization problem can be written as

$$
\begin{array}{cc}
\min _{I, T_{s}^{(i)}, N^{(i)}} \varepsilon=R_{t}\left(I, T_{s}^{(i)}, N^{(i)}\right)-D_{t} \\
\text { s.t. } \quad 0 \leq T_{s}^{(i)} \leq T_{f}, \quad \forall i \\
\left(T_{s}^{(i)}+N^{(i)} T_{s r}\right) \leq T_{f}, \quad \forall i \\
Q_{d}^{(i)} \geq \beta^{(i)}, \quad \forall i \\
\varepsilon \geq 0 \\
1 \leq I \leq I_{\max }
\end{array}
$$

where $T_{s}^{(i)}$ and $N^{(i)}$ are respectively the spectrum sensing duration and the number of cooperating nodes involved in CSS for channel $i ; I_{\max }$ is the maximum number of channels available to be exploited; $Q_{d}^{(i)}$ is the cooperative probability of detection for channel $i$ and $\beta^{(i)}$ is a lower bound on this. The constraint $\varepsilon \geq 0$ is included to ensure that the throughput demand is met.

It is shown in [16] that the optimal solution for (23) can be achieved when constraint $Q_{d}^{(i)} \geq \beta^{(i)}, \forall i$ is satisfied with equality. When this constraint is at equality and for a chosen fusion threshold $K$ (in this paper, we consider $K=\left\lceil\frac{N}{2}\right\rceil$ ), the corresponding device's probability of detection $P_{d}$ can be found from $Q_{d}^{(i)}$ using Eq. (10). Notice that to use this equation, it is required that the probability of detection $P_{d}$ is the same for all sensing devices, while the effect of different $\mathrm{SNR}_{p}$ is absorbed by the device's probability of false alarm $P_{f}$ (c.f. Eq. (5)). Although the simplified Eq. (10) is used to find the probability of detection $P_{d}$, the general Eq. (9) is used to calculate the cooperative false alarm $Q_{f}$. In addition, notice that finding the optimal $T_{s}$ and $N$ is equivalent to finding the optimal $\alpha$ (i.e., maximizing $\alpha$ will minimize $T_{s}$ and $N$ ); hence, we also can write (23) as

$$
\begin{gathered}
\min \varepsilon\left(I, \alpha\left(T_{s}^{(i)}, N^{(i)}\right)\right) \\
\text { s.t., } \quad 0 \leq T_{s}^{(i)} \leq T_{f}, \forall i \\
\left(T_{s}^{(i)}+N^{(i)} T_{s r}\right) \leq T_{f}, \forall i \\
\varepsilon>0 \\
1 \leq I \leq I_{\max } \\
0 \leq \alpha\left(T_{s}^{(i)}, N^{(i)}\right) \leq 1, \forall i
\end{gathered}
$$


For this optimization problem, we propose to find the solution by using a two-dimensional search method.

\section{Numerical analysis}

In this section, we numerically analyze the performance of the TD-CRHAN and compare it with the conventional solution. For this section, let us assume that the $\mathrm{SNR}_{p_{j}}^{(i)}=$ $\mathrm{SNR}_{p}$ and $P\left(\mathcal{H}_{0}^{(i)}\right)=P\left(\mathcal{H}_{0}\right)$, i.e., are the same, for all $i$ and $j$, and we note that these parameters will be randomized based on a uniform distribution during the simulation analysis (Section 5). The following values are considered and fixed throughout this section in which most of them are also used in [6]: $T_{f}=105 \mu \mathrm{s}, T_{s r}=4 \mu \mathrm{s}, \beta^{(i)}=0.9$ and $W^{(i)}=5 \mathrm{MHz}$, for all $i$. Moreover, in this work, the majority fusion rule is considered for the CSS as this has been found to be optimal or nearly optimal $[5,6,17]$. For the solution of the optimization problem, we consider $T_{s}^{(i)}=T_{s}$ and $N^{(i)}=N$ for all $i$.

Graphs of $\varepsilon$ versus the total number of in-band channels $I$ and a) the data transmission time coefficient $\alpha$, and b) the duration required for local spectrum sensing $T_{s}$, are shown in Fig. 6a, b, respectively, where $\mathrm{SNR}_{p}=-7$ $\mathrm{dB}, P\left(\mathcal{H}_{0}\right)=0.7, N=6$, and $D_{t}=3.5 \mathrm{Mb} / \mathrm{s}$. Note

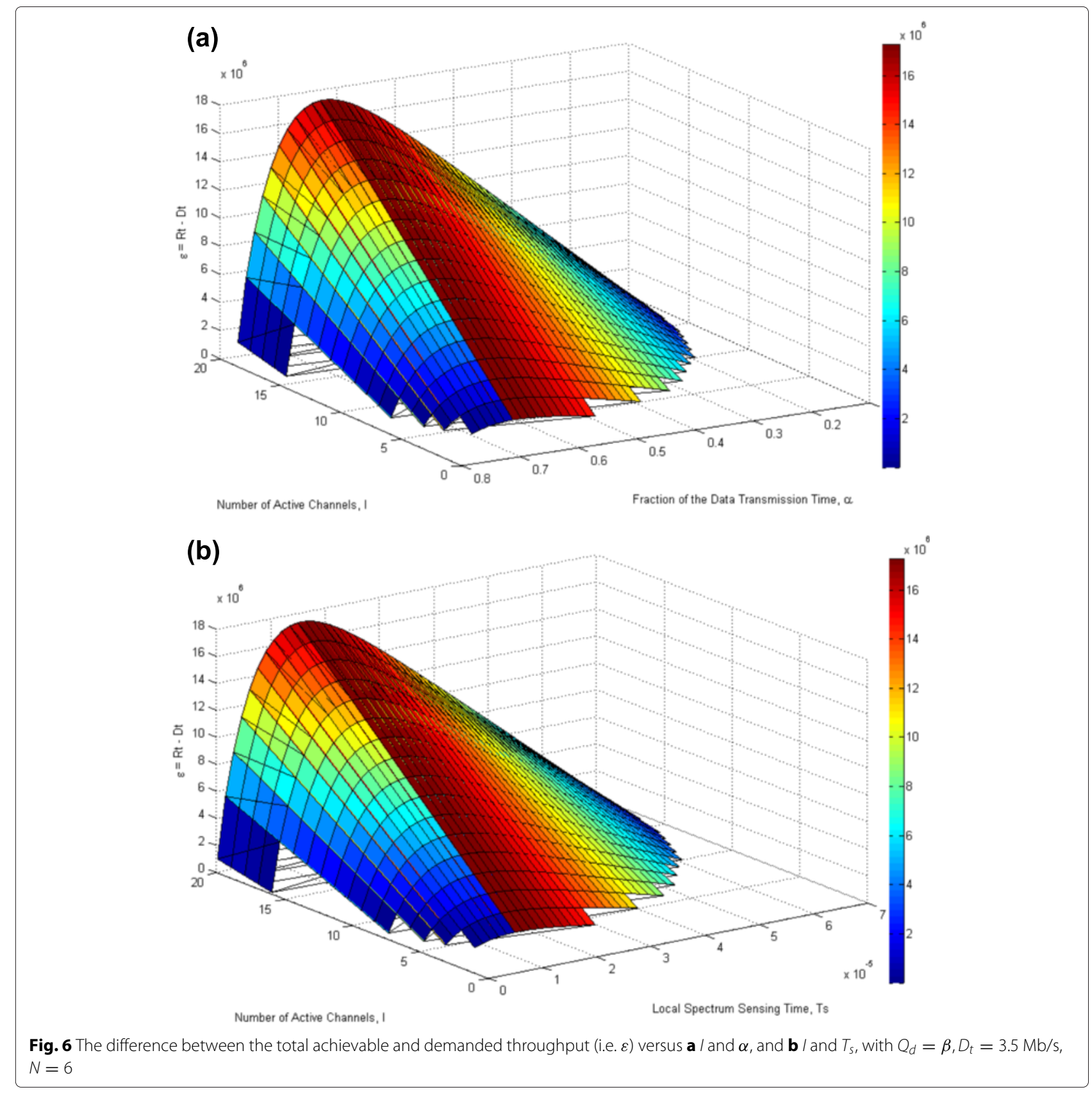



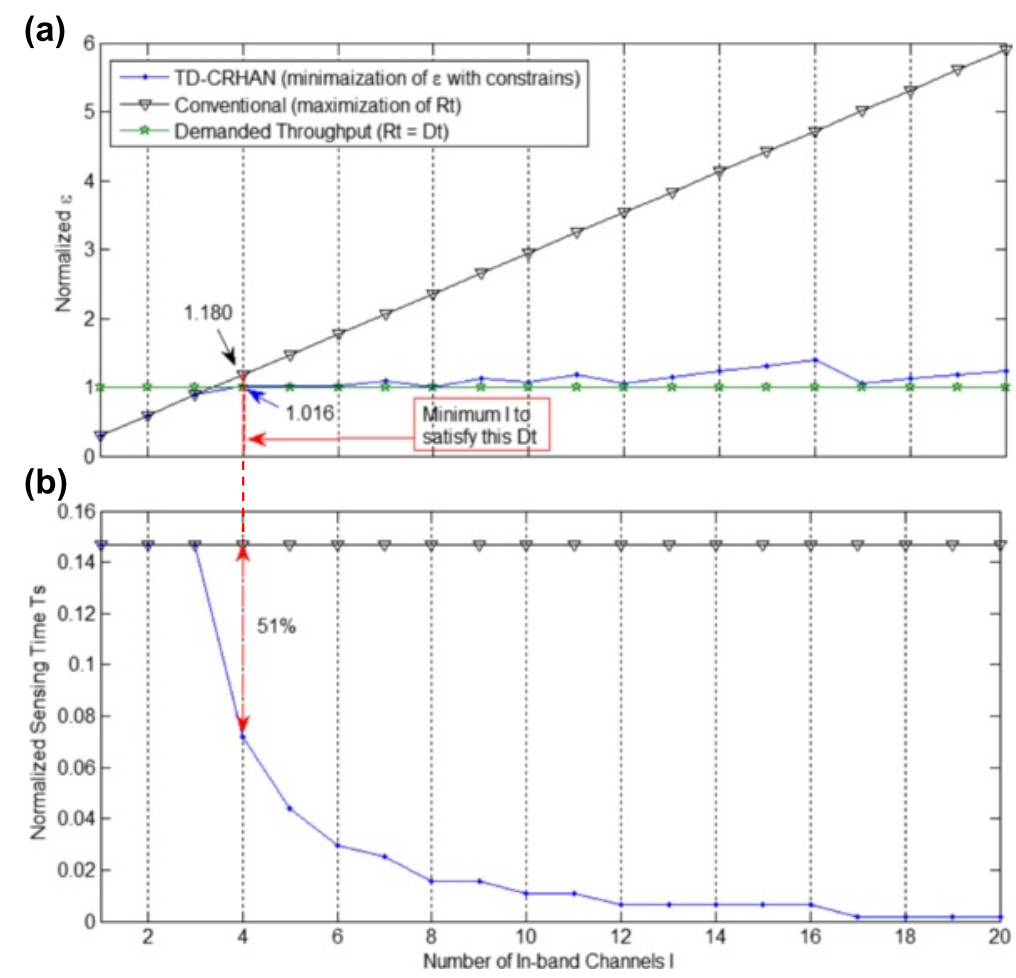

Fig. 7 Effects of different total number of in-band channels / on $\mathbf{a}$ normalized $\varepsilon=\frac{\varepsilon}{D_{t}}$, and $\mathbf{b}$ fraction of time used for spectrum sensing (i.e., normalized sensing time $=\frac{T_{s}}{T_{f}}$ ). This is a comparison between TD-CRHAN and the conventional scheme. For this, $D_{t}$ is fixed at $3.5 \mathrm{Mb} / \mathrm{s}$ and $\mathrm{N}=6$
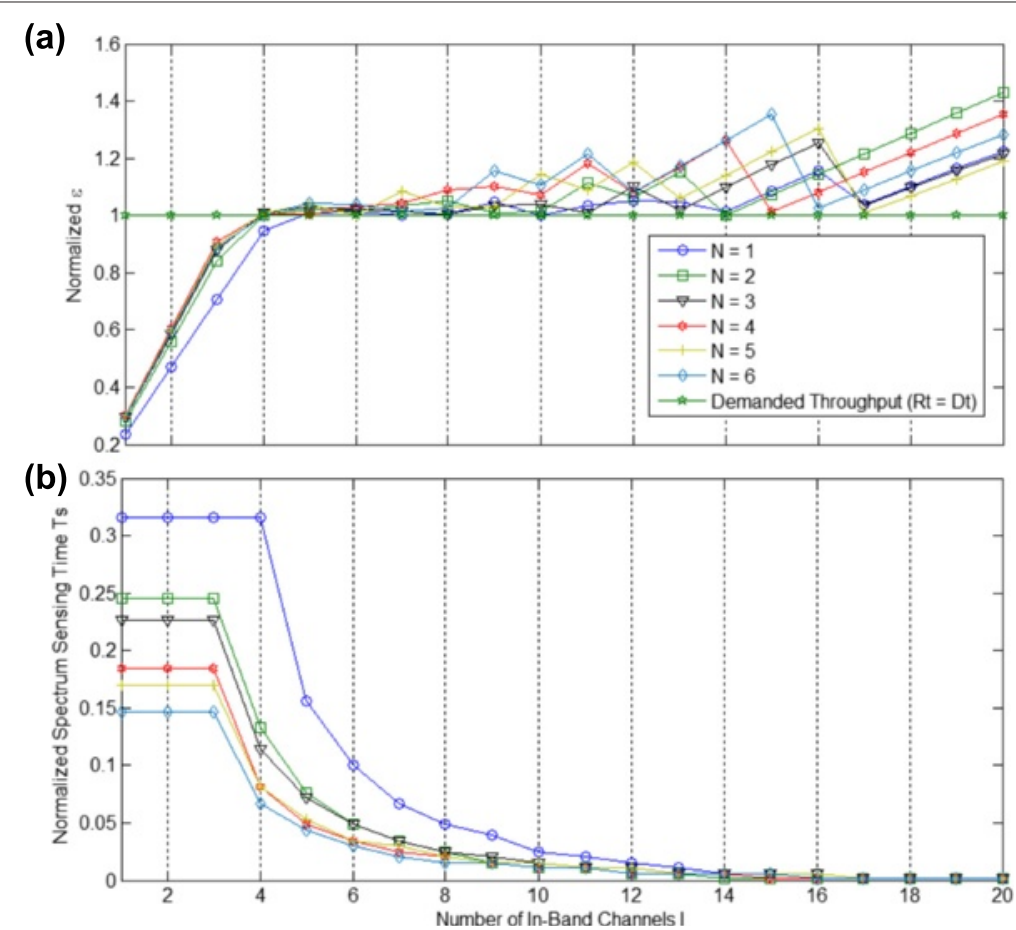

Fig. 8 Impact of varying number of cooperating sensing devices $N$ on $\mathbf{a}$ normalized $\varepsilon=\frac{\varepsilon}{D_{t}}$, and $\mathbf{b}$ fraction of time used for spectrum sensing (i.e., normalized sensing time $=\frac{T_{s}}{T_{f}}$ ). For this, $D_{t}=3.5 \mathrm{Mb} / \mathrm{s}$ 


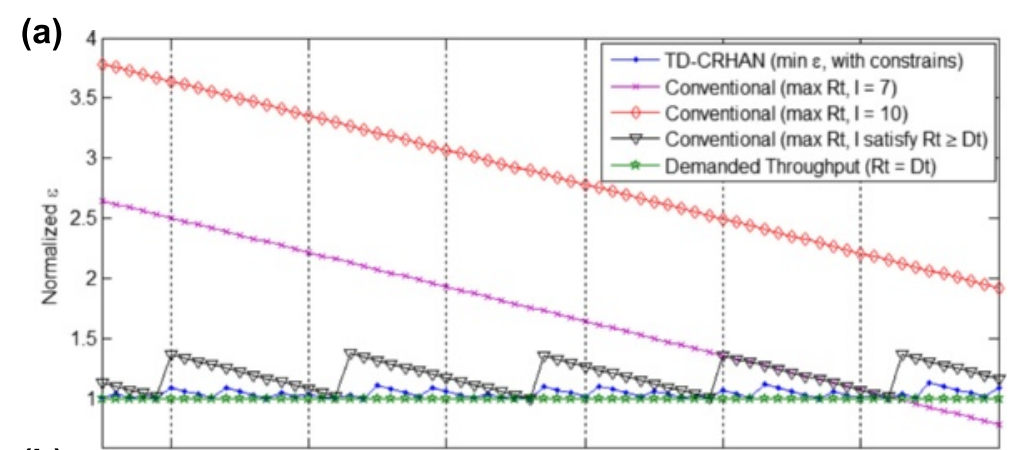

(b)
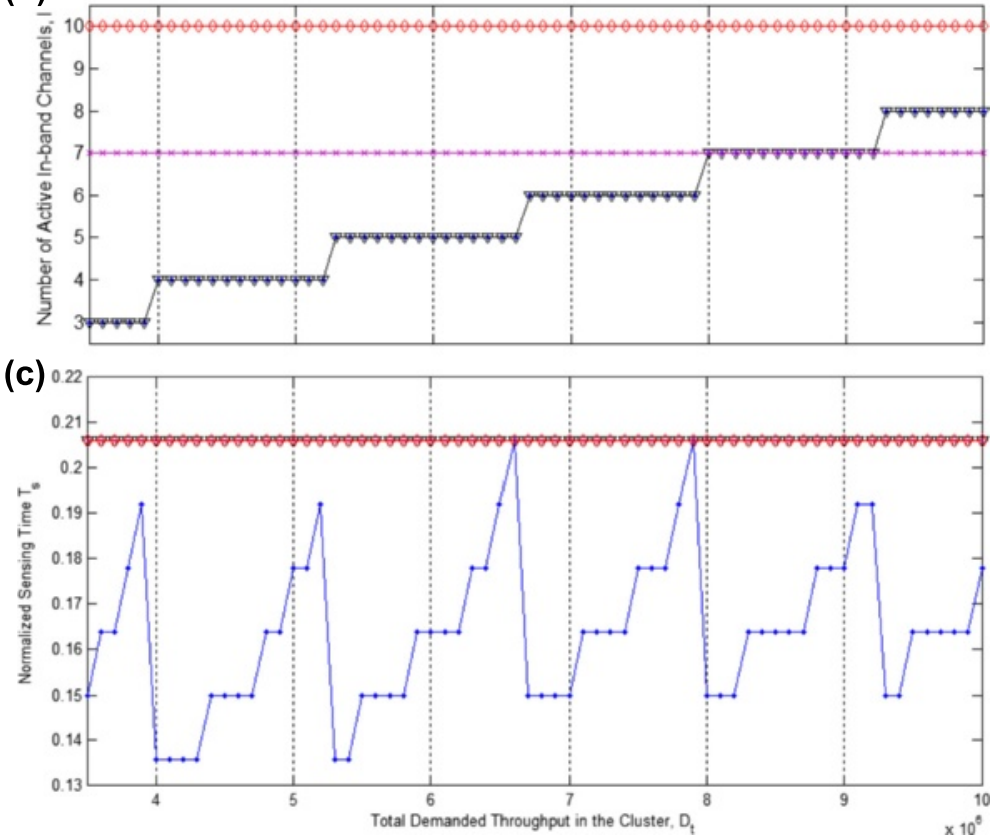

Fig. 9 Effects of different cluster's throughput demand, $D_{t}$ on $\mathbf{a}$ normalized $\varepsilon=\frac{\varepsilon}{D_{t}}$, $\mathbf{b}$ number of in-band channels / used, and $\mathbf{c}$ fraction of time used for spectrum sensing (i.e., normalized sensing time $=\frac{T_{s}}{T_{f}}$ ). This is a comparison between TD-CRHAN and the conventional schemes. For this, we take $\mathrm{SNR}_{p}=-7 \mathrm{~dB}$ and $\mathrm{N}=6$

that all points on the graphs satisfy every constraint given in (24). It is seen that for each value of $I, \varepsilon\left(T_{s}\right)$ and $\varepsilon(\alpha)$ are both concave functions in which the peak points of these functions are the maximum achievable throughput of the cluster. In the conventional CR, these points are considered as optimal. However, it is seen from these graphs that there is an excess throughput (i.e., $\varepsilon>0$ ), which is then not going to be used by the network. This throughput underutilization becomes larger with increasing the number of in-band channels $I$. In contrast, TD-CRHAN tries to find the lowest point of this graph which is the minimum possible $\varepsilon$ and at the same time satisfies all the constraints listed in (24). By doing this, TDCRHAN can relax the required local spectrum sensing time $T_{S}$ and the number of cooperating nodes $N$ of the CR system.
From Fig. 6, the optimal points of the conventional and the proposed TD-CRHAN are taken out and the normalized $\varepsilon$ is plotted in Fig. $7 \mathrm{a}$, and the corresponding normalized sensing time $T_{s}$ (i.e., fraction of time used for spectrum sensing in a frame) is plotted in Fig. 7b. It is seen that $\varepsilon$ is linearly proportional to the number of inband channels $I$ for the conventional case. This is because the total achievable throughput $R_{t}$ for this case is equal to the maximum achievable throughput of each channel multiplied by the total number of in-band channels, i.e., $R_{t}=R \cdot I$ (due to the above assumptions, we have $R^{(i)}=$ $R, \forall i)$; hence, the larger $I$, the higher $\varepsilon$ irrespective of the $D_{t}$. On the other hand, in a TD-CRHAN, $R_{t}$ is adjusted as near as possible to $D_{t}$, which is actually the minimization of $\varepsilon$ with constraints. As a result, it can be seen that with a TD-CRHAN scheme, $\varepsilon$ is maintained as low as possible, 
and the spectrum sensing time $T_{s}$ is significantly relaxed as compared to the conventional scheme (as shown in Fig. 7b). These gains become larger as $I$ becomes higher. It is also denoted in Fig. 7a that the minimum $I$ required to satisfy $D_{t}$ is 4 . Projecting this point to Fig. $7 \mathrm{~b}$ (as depicted by the red arrows) shows that, even at this point, TDCRHAN obtains around $51 \%$ gain on the required $T_{s}$ in comparison with the conventional case.

Further, the impact of the number of cooperative sensing devices $N$ on the proposed TD-CRHAN is analyzed as depicted in Fig. 8. In general, it can be seen that, the higher $N$, the lesser the $T_{s}$ required, which means a higher $N$ will reduce the burden of sensing on the individual CR-based HAN device. However, it can be noticed that $T_{s}$ is saturated and then becomes constant after a certain $I$ (in this case $I>6$ ); thus, at this point, an increase of $N$ or $I$ would not reduce $T_{s}$ anymore, hence it will increase the value of $\varepsilon$ as witnessed in Fig. 7a.

Next, with the same setting, we analyze the performance of the proposed TD-CRHAN in comparison with the conventional one for different $D_{t}$. Three scenarios of the conventional settings are considered: 1) maximization of $R_{t}$ with $\left.I=7,2\right)$ maximization of $R_{t}$ with $I=10$, and 3) maximization of $R_{t}$ with $I$ is set based on the network throughput demand $D_{t}$ such that $R_{t} \geq D_{t}$. Figure 9a shows that the TD-CRHAN scheme satisfies the throughput demand at all times and has least throughput underutilization compared to other schemes, in particular for the cases that $R$ is maximized without $D_{t}$ consideration. Worse, the conventional plot without $D_{t}$ consideration (i.e., scenarios 1 and 2) are unable to satisfy the demanded throughput after a certain point (for instance, in this case:

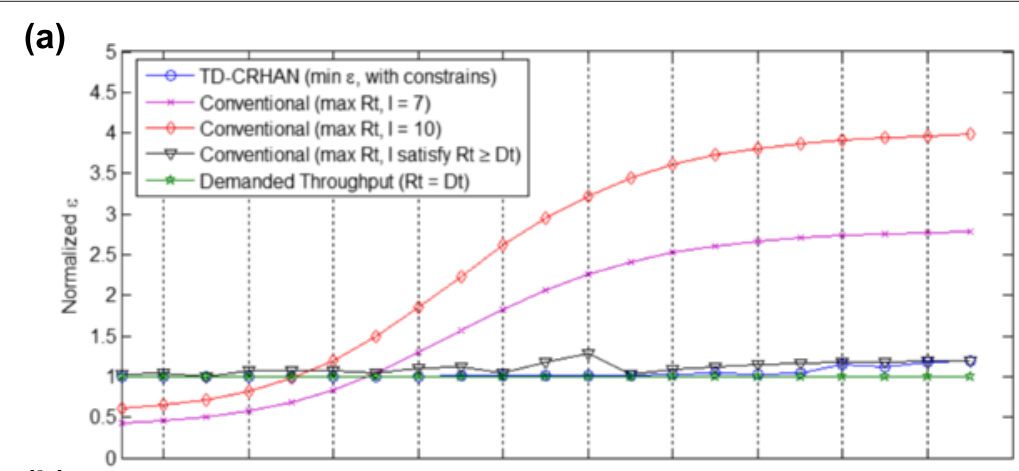

(b)

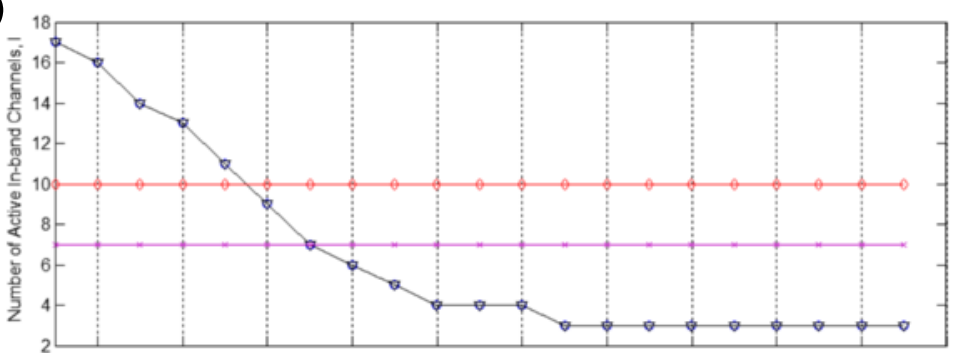

(c) 0

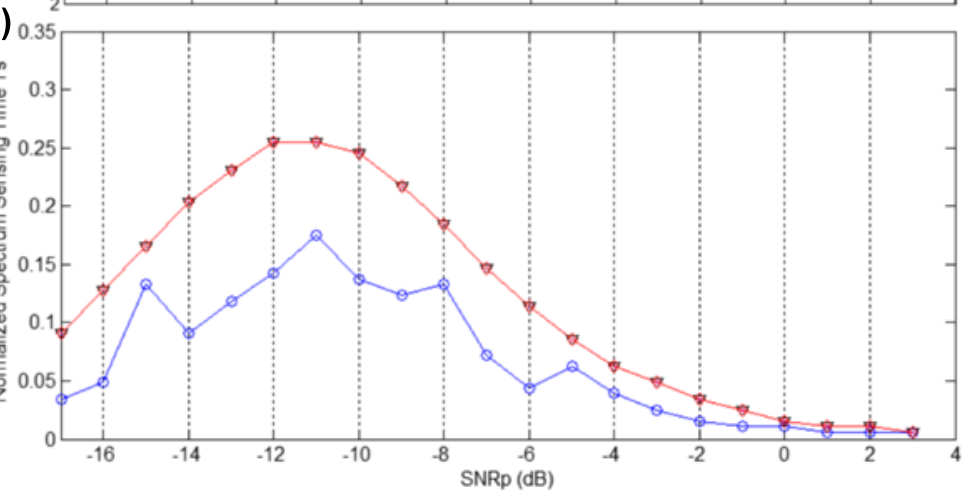

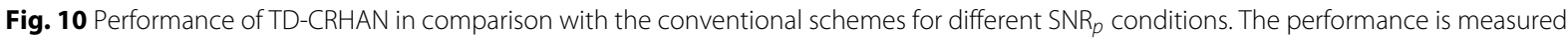
in term of a normalized $\varepsilon=\frac{\varepsilon}{D_{t}}$, b number of in-band channels / used, and c fraction of time used for spectrum sensing (i.e., normalized sensing time $\left.=\frac{T_{s}}{T_{f}}\right)$, with $P\left(\mathcal{H}_{0}\right)$ fixed at 0.7 
scenario 1 could not satisfy the demand for $D_{t}>9.3 \mathrm{Mb} / \mathrm{s}$ as the number of in-band channels is fixed to 7). In contrast, in principle the proposed TD-CRHAN can support an unlimited $D_{t}$ if $I$ is unlimited.

We then numerically analyze the impact of the channel conditions, i.e., $\mathrm{SNR}_{p}$ and $P\left(\mathcal{H}_{0}\right)$, on the performance of TD-CRHAN as well as the three conventional schemes of which the results are shown in Figs. 10 and 11, respectively. It is witnessed that, for the proposed TD-CRHAN scheme, $D_{t}$ will always be satisfied at minimum $\varepsilon$ for almost any $\mathrm{SNR}_{p}$ or $P\left(\mathcal{H}_{0}\right)$ values (as shown in Figs. 10a and 11a). This is because, a TD-CRHAN allows for an adaptive number of active in-band channels $I$ (refer to Figs. 10b and 11b) and local spectrum sensing duration $T_{s}$ (refer to Figs. 10c and 11c) where these values are optimized such that the resultant achievable throughput $R_{t}$ is very close to the corresponding demand $D_{t}$. Specifically, for $I$, at a very low $\mathrm{SNR}_{p}$ or $P\left(\mathcal{H}_{0}\right)$, its value will be increased while it will be reduced to the minimal at a high $\mathrm{SNR}_{p}$ or $P\left(\mathcal{H}_{0}\right)$. Notice that $D_{t}$ is still satisfied even for $P\left(\mathcal{H}_{0}\right)=0$ in which the network throughput at this point is acquired from the $P\left(\mathcal{H}_{1}\right)$ part (i.e., at the expense of a high $I$ ). For $T_{s}$, it is seen in Fig. 10c that it is adjusted to a lower value at a very low $\mathrm{SNR}_{p}$. This is because at this point, $\varepsilon$ (and $R_{t}$ ) is influenced more by $T_{s}$ but less by $P_{f}$ (and $Q_{f}$ ) as at a very low $\mathrm{SNR}_{p}$, a high $T_{s}$ does not provide a significant reduction to $P_{f}$ (i.e., this can be seen from (5), as plotted in Fig. 12). On such a case, a lower $T_{s}$ is more favorable in order to satisfy the demanded throughput $D_{t}$ and meet the $\varepsilon \geq 0$ constraint. However, $P_{f}$ (and $Q_{f}$ ) become more dominant with the increase of $\mathrm{SNR}_{p}$ up to a certain point, but yet it is dominated by $T_{s}$ when $P_{f}$ becomes saturated; this can be observed in Fig. 10c. Similarly with Fig. 11c, that is at a very low $P\left(\mathcal{H}_{0}\right), T_{s}$ will be set to a lower value as a high $T_{s}$ is not beneficial because at this instance most of the $R_{t}$

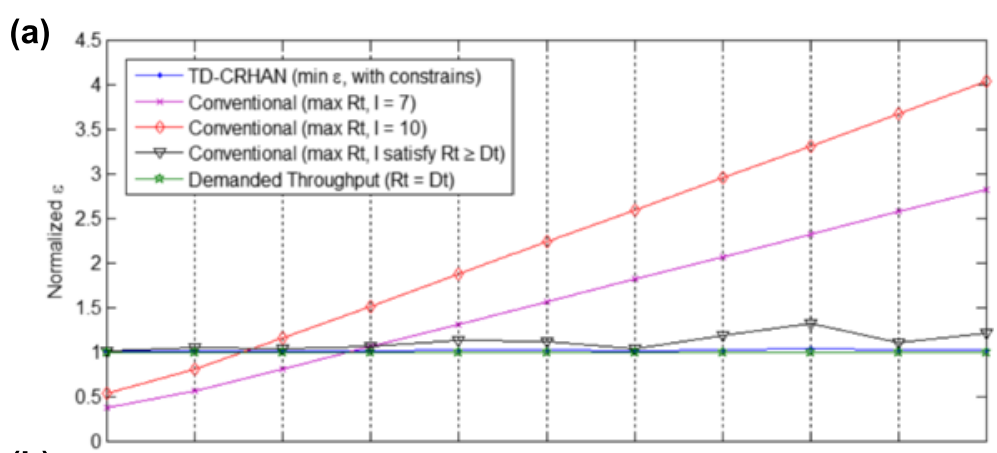

(b)
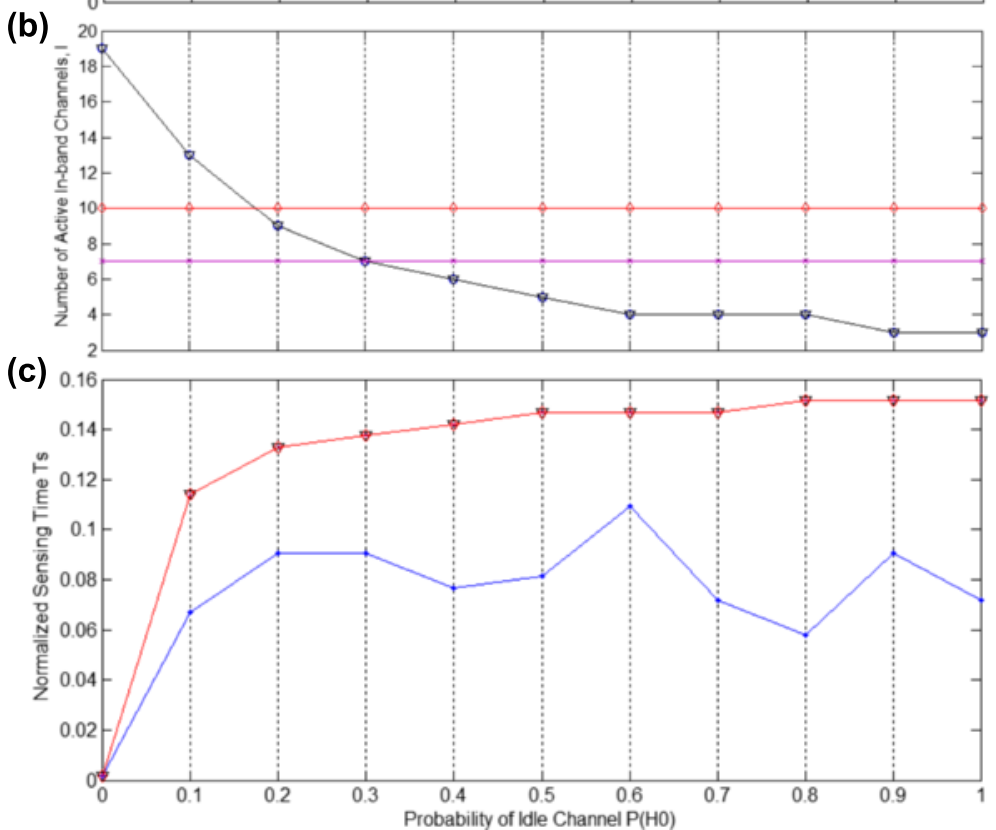

Fig. 11 Performance of TD-CRHAN in comparison with the conventional schemes for different $P\left(\mathcal{H}_{0}\right)$ conditions. The performance is measured in term of $\mathbf{a}$ normalized $\varepsilon=\frac{\varepsilon}{D_{t}}$, $\mathbf{b}$ number of active in-band channels / used, and $\mathbf{c}$ fraction of time used for spectrum sensing (i.e., normalized sensing time $\left.=\frac{T_{s}}{T_{f}}\right)$, with $S N R_{p}$ fixed at $-7 \mathrm{~dB}$ 


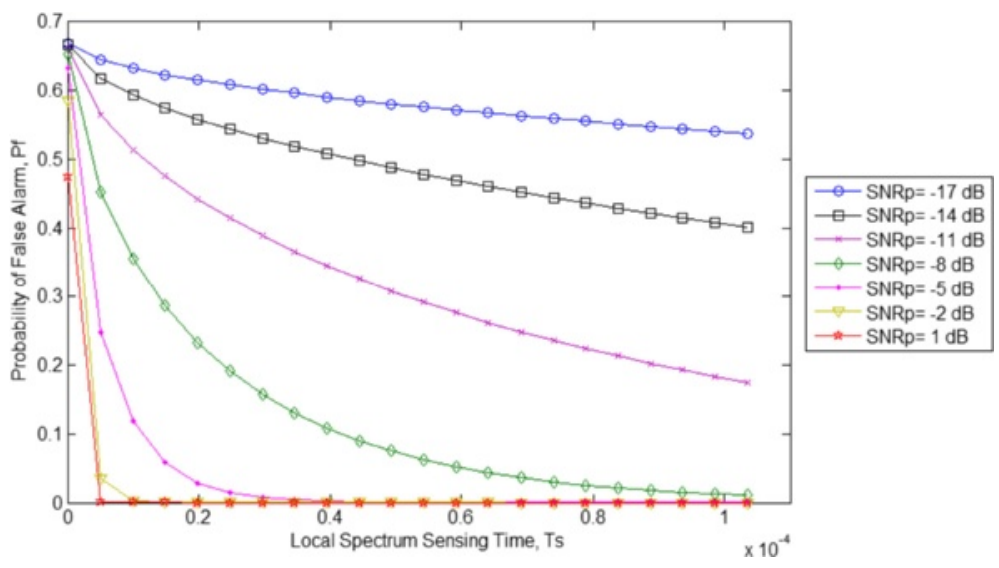

Fig. $12 P_{f}$ versus $T_{s}$ for different $S N R_{p}$ with $P_{d}$ fixed at 0.9

comes from $P\left(\mathcal{H}_{1}\right)$ (c.f., (19)). Note that in (19) a higher $T_{s}$ leads to a lower $Q_{f}$ and therefore a higher $R_{t}$; at a very low $P\left(\mathcal{H}_{0}\right)$, a lower $Q_{f}$ does not help because this part of $R_{t}$ is suppressed by the value of $P\left(\mathcal{H}_{0}\right)$ itself, and vice versa.

\section{Simulation results and analysis}

In this section, we run Monte Carlo simulations on the proposed TD-CRHAN scheme and the three conventional cases and compare the results with the numerical results. The settings for this simulation are the same as in Section 4 except the SNR of the incumbent user (i.e., $\mathrm{SNR}_{p_{j}}$ ) is randomly set for each CR-based device $j$ based on the Uniform distribution within the range of -11 and $3 \mathrm{~dB}$ (i.e., $\mathrm{SNR}_{p_{j}} \sim \mathcal{U}(-11,3) \mathrm{dB}$, for all $j$ ). We consider this range for this simulation in order to capture the dynamic behavior of the sensing qualities (i.e., $P_{d}$ and $\left.P_{f}\right)$ and observe the impact of different sensing

(a)

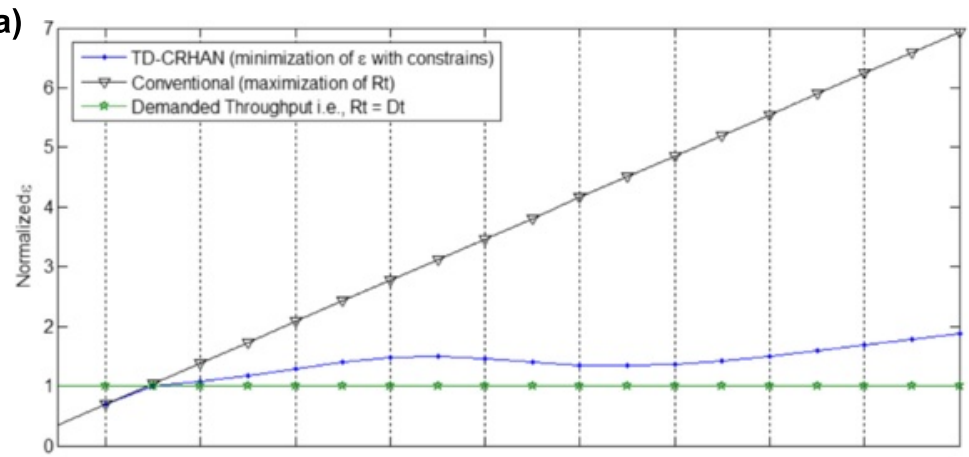

(b)

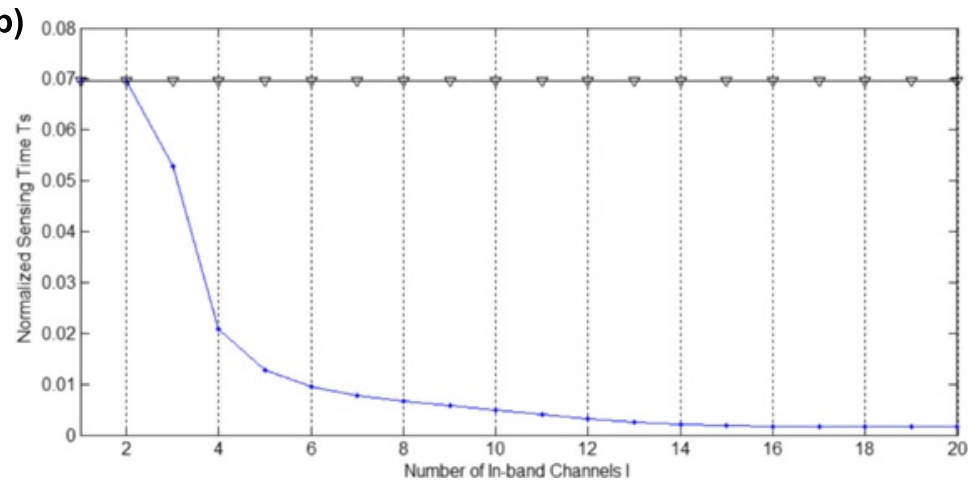

Fig. 13 Simulation results on the effects of using different total number of in-band channels / on a normalized $\varepsilon=\frac{\varepsilon}{D_{t}}$, and $\mathbf{b}$ fraction of time used for spectrum sensing (i.e., normalized sensing time $=\frac{T_{s}}{T_{f}}$ ). This is a comparison between TD-CRHAN and the conventional schemes. For this, SNR $p_{j}$ is chosen randomly between -11 and $3 \mathrm{~dB}$ for all $j$ 
time $T_{s}$ values. For $\mathrm{SNR}_{p}$ higher than $3 \mathrm{~dB}$, only a few samples with single device sensing (without cooperation) are required to obtain an already very high probability of detection $P_{d}$ and very low probability of false alarm $P_{f}$. For low $\mathrm{SNR}_{p}$ used, i.e., $-11 \mathrm{~dB}$ and less, an increase of the sensing time does not really give significant improvement of the sensing qualities. We repeat the simulation 1000 times and the results are averaged. It can be seen from Fig. 13 that, in general, the patterns of the simulation results are similar with the graphs from the numerical analysis (refer to Fig. 7). However, notice that the optimal sensing time in the simulation is less than in the numerical analysis which is caused by the possible high value of the incumbent user signal strength in the simulation (i.e., between -11 and $3 \mathrm{~dB}$ as compared to a fix $-7 \mathrm{~dB}$, respectively). In addition, Fig. 14 shows that the corresponding cooperative and the individual false alarm probabilities, i.e., $Q_{f}$ and $P_{f_{j}}, \forall j$, respectively, of the proposed TD-CRHAN varies according to the number of in-band channels $I$ available. In TD-CRHAN, for the same total throughput demand $D_{t}$, an increase of the number of in-band channels $I$ will decrease the required achievable throughput $R^{(i)}$ of each channel $i$, hence this reduces the required $Q_{f}$ and the corresponding $P_{f_{j}}, \forall j$. This then further reduces the required sensing time $T_{s}$, as can be seen in Fig. 13. Besides, it can be observed from Fig. 14a that the probability of false alarm $P_{f_{j}}$ of sensing device $j$ depends on its $\mathrm{SNR}_{p_{j}}$ : a lower $\mathrm{SNR}_{p_{j}}$ device has a higher $P_{f_{j}}$.

Finally, a Monte Carlo simulation is executed in which all network parameters are uniformly randomized (i.e., $D_{t} \sim \mathcal{U}(3.5,10) \mathrm{Mb} / \mathrm{s}, \mathrm{SNR}_{p_{j}}^{(i)} \sim \mathcal{U}(-11,3) \mathrm{dB}$ and $\left.P\left(\mathcal{H}_{0}^{(i)}\right) \sim \mathcal{U}(0,1), \forall i, j\right)$ to evaluate the performance of the TD-CRHAN in a more practical scenario. The graphs of the normalized $\varepsilon$ and sensing time $T_{s}$, versus the number of in-band channel $I$ for $N=1,2, \ldots, 6$ are plotted as shown in Fig. 15. Similarly, it is observed that with the proposed TD-CRHAN scheme, the network throughput demand $D_{t}$ is satisfied at all times for all $N$. However, it is seen that a lower number of cooperating sensing devices $N$ will require a higher sensing time $T_{s}$, and moreover at a certain point, a higher number of channels $I$ is even required (i.e., in this case, $I \geq 4$ for $N=1$ and 2 compared to $I \geq 3$ for $N=3,4,5$, and 6).

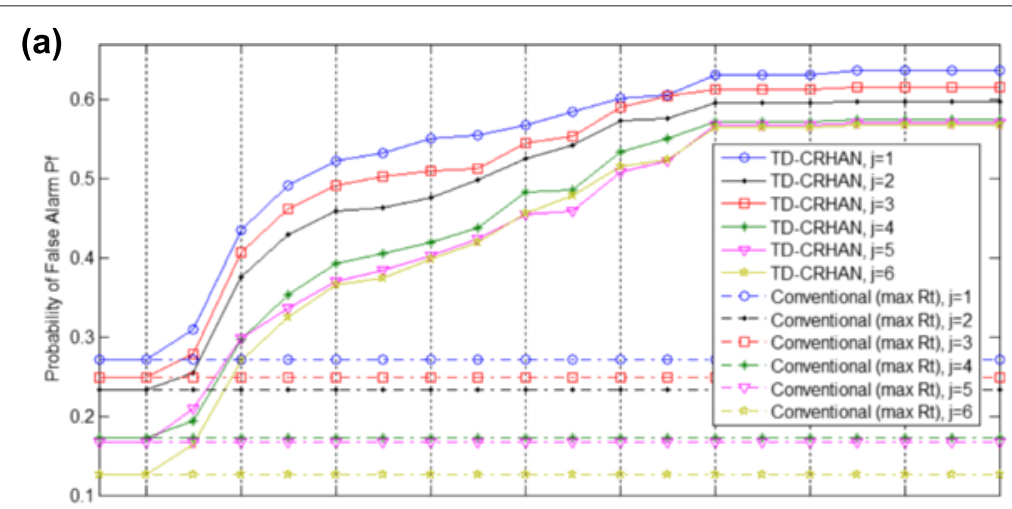

(b)

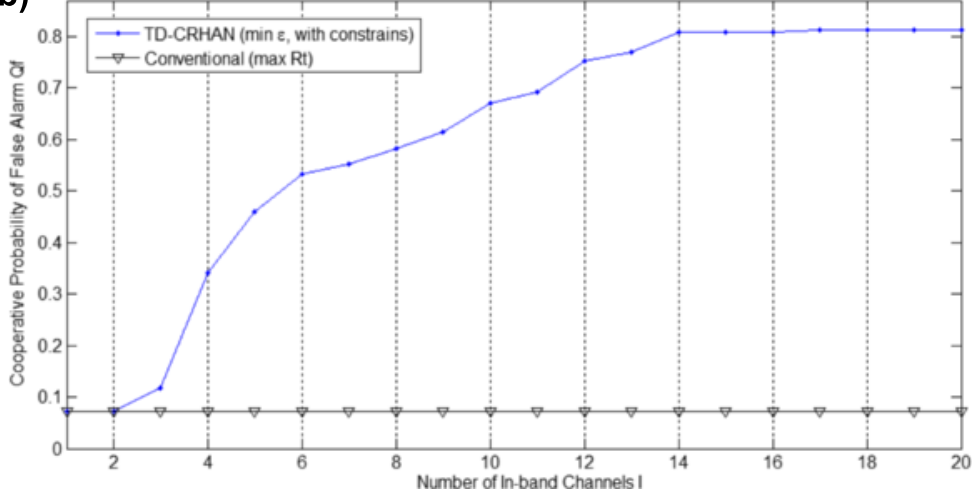

Fig. 14 The corresponding a false alarm probability $P_{f_{j}}$ of each device $j$, and $\mathbf{b}$ cooperative false alarm probability $Q_{f}$, from the simulation of which the incumbent $S N R_{p_{j}}$ are different at each sensing device $j$. In the simulation, the random generated $S N R_{p_{j}}, \forall j$ are as the following: $S N R_{p_{j}}=\{-6.5 \mathrm{~dB}$, $-5.3 \mathrm{~dB},-5.9 \mathrm{~dB},-3.9 \mathrm{~dB},-3.5 \mathrm{~dB},-3.2 \mathrm{~dB}\}$ for $j=\{1, \ldots, 6\}$, respectively 

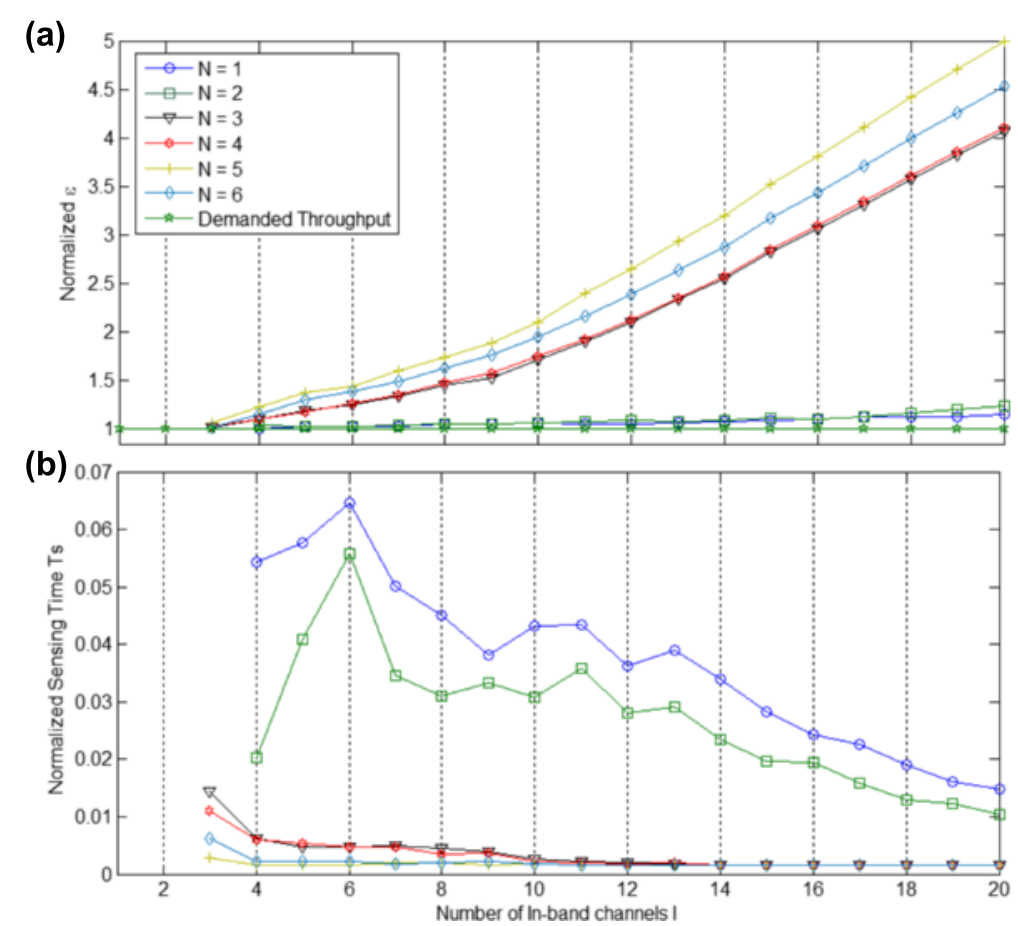

Fig. 15 Simulation results of the total number of active channels / versus: a normalized $\varepsilon=\frac{\varepsilon}{D_{t}}$, and $\mathbf{b}$ fraction of time used for spectrum sensing (i.e., normalized sensing time $=\frac{T_{s}}{T_{f}}$ ). This is for different number of cooperating devices i.e., $N=1,2, \cdots, 6$ with more practical scenario in which all network parameters are randomly chosen, i.e., $D_{t} \sim \mathcal{U}(3.5,10) \mathrm{Mb} / \mathrm{s}, \mathrm{SNR}_{p_{j}}^{(i)} \sim \mathcal{U}(-11,3) \mathrm{dB}$ and $P\left(\mathcal{H}_{0}\right) \sim \mathcal{U}(0,1)$, for all $i$ and $j$

\section{Conclusions}

To support the ever-rising throughput demand of home area networks (HAN), we proposed in this paper a cognitive radio (CR)-based communication scheme called TD-CRHAN. The TD-CRHAN aims at satisfying the demanded network throughput with equality by determining the optimal local spectrum sensing time, the number of cooperating sensing devices, and the number of active in-band channels needed. This leads to an efficient scheme which provides a higher utilization of the occupied channels. It was shown by extensive numerical analysis and through simulations that TDCRHAN is able to relax the tight cooperative spectrum sensing requirements and provides significant gains on the cooperative spectrum sensing parameters (i.e., spectrum sensing time and number of cooperating devices), compared to the conventional solution. More specifically, TD-CRHAN reduces the required local spectrum sensing time by more than $51 \%$. Furthermore, it was shown that these cooperative spectrum sensing parameters can be further improved with the availability of additional cooperating devices or channels (bandwidth).

\section{Competing interests}

The authors declare that they have no competing interests.

\section{Acknowledgements}

The work has been supported partially by the Ministry of Education, Malaysia.

Received: 18 September 2014 Accepted: 11 September 2015

Published online: 02 October 2015

\section{References}

1. M Nekovee, A survey of cognitive radio access to tv white spaces. Int. J. Digit. Multimed. Broadcast. 2010(236568) (2010). doi:10.1155/2010/ 236568

2. Y-C Liang, Y Zeng, ECY Peh, AT Hoang, Sensing-throughput tradeoff for cognitive radio networks. IEEE Trans. Wirel. Commun. 7(4), 1326-1337 (2008). doi:10.1109/TWC.2008.060869

3. L Tan, L Le, Joint cooperative spectrum sensing and MAC protocol design for multi-channel cognitive radio networks. EURASIP J. Wirel. Commun. Netw. 2014(1), 101 (2014). doi:10.1186/1687-1499-2014-101

4. G Umashankar, AP Kannu, Throughput optimal multi-slot sensing procedure for a cognitive radio. IEEE Commun. Lett. 17(12), 2292-2295 (2013). doi:10.1109/LCOMM.2013.102613.131825

5. RA Rashid, A. H. F. A. Hamid, N Fisal, MA Sarijari, RA Rahim, A Mohd, in 2012 IEEE Symposium on Wireless Technology and Applications (ISWTA). Optimal user selection for decision making in cooperative sensing, (2012), pp. 165-170. doi:10.1109/ISWTA.2012.6373834

6. S Maleki, SP Chepuri, G Leus, Optimization of hard fusion based spectrum sensing for energy-constrained cognitive radio networks. Phys. Commun. 9(0), 193-198 (2013). doi:10.1016/j.phycom.2012.07.003

7. M Najimi, A Ebrahimzadeh, SMH Andargoli, A Fallahi, A novel sensing nodes and decision node selection method for energy efficiency of cooperative spectrum sensing in cognitive sensor networks. IEEE Sensors J. 13(5), 1610-1621 (2013). doi:10.1109/JSEN.2013.2240900

8. H Kim, KG Shin, Efficient discovery of spectrum opportunities with MAC-layer sensing in cognitive radio networks. IEEE Trans Mob Comput. 7(5), 533-545 (2008). doi:10.1109/TMC.2007.70751 
9. K-LA Yau, N Ramli, W Hashim, H Mohamad, Clustering algorithms for cognitive radio networks: A survey. J. Netw. Comput. Appl. 45(0), 79-95 (2014). doi:10.1016/j.jnca.2014.07.020

10. IEEE Standard for information technology- local and metropolitan area networks- specific requirements- part 22: Cognitive wireless RAN medium access control (MAC) and physical layer (PHY) specifications: Policies and procedures for operation in the TV bands, 1-680 (2011). IEEE Std 802.22-2011, doi:10.1109/IEEESTD.2011.5951707

11. M Timmers, S Pollin, A Dejonghe, L Van der Perre, F Catthoor, A distributed multichannel MAC protocol for multihop cognitive radio networks. IEEE Trans. Veh. Technol. 59(1), 446-459 (2010). doi:10.1109/TVT.2009.2029552

12. SChaudhari, J Lunden, V Koivunen, HV Poor, Cooperative sensing with imperfect reporting channels: Hard decisions or soft decisions? IEEE Trans. Sig. Process. 60(1), 18-28 (2012). doi:10.1109/TSP.2011.2170978

13. R Rajbanshi, AM Wyglinski, GJ Minden, in 1st International Conference on Cognitive Radio Oriented Wireless Networks and Communications. An efficient implementation of NC-OFDM transceivers for cognitive radios, (2006), pp. 1-5. doi:10.1109/CROWNCOM.2006.363452

14. VRS Banjade, N Rajatheva, in 8th International Symposium on Wireless Communication Systems (ISWCS). Primary user capacity maximization in cooperative detection network using $m$ out of $\mathrm{n}$ fusion rule, (2011), pp. 482-486. doi:10.1109/ISWCS.2011.6125406

15. YH Wang, On the number of successes in independent trials. Stat. Sin. 3(2), 295-312 (1993)

16. ECY Peh, Y-C Liang, YL Guan, Y Zeng, Optimization of cooperative sensing in cognitive radio networks: A sensing-throughput tradeoff view. IEEE Trans. Veh. Technol. 58(9), 5294-5299 (2009). doi:10.1109/TVT. 2009.2028030

17. W Zhang, RK Mallik, K Letaief, Optimization of cooperative spectrum sensing with energy detection in cognitive radio networks. IEEE Trans. Wirel. Commun. 8(12), 5761-5766 (2009). doi:10.1109/TWC.2009. 12.081710

\section{Submit your manuscript to a SpringerOpen ${ }^{\mathcal{O}}$ journal and benefit from:}

- Convenient online submission

- Rigorous peer review

- Immediate publication on acceptance

- Open access: articles freely available online

- High visibility within the field

- Retaining the copyright to your article

Submit your next manuscript at $\boldsymbol{\wedge}$ springeropen.com 